MATHEMATICS OF COMPUTATION

Volume 77, Number 261, January 2008, Pages 71-92

S 0025-5718(07)02019-4

Article electronically published on May 14, 2007

\title{
ADAPTIVE WAVELET ALGORITHMS FOR ELLIPTIC PDE'S ON PRODUCT DOMAINS
}

\author{
CHRISTOPH SCHWAB AND ROB STEVENSON
}

\begin{abstract}
With standard isotropic approximation by (piecewise) polynomials of fixed order in a domain $D \subset \mathbb{R}^{d}$, the convergence rate in terms of the number $N$ of degrees of freedom is inversely proportional to the space dimension $d$. This so-called curse of dimensionality can be circumvented by applying sparse tensor product approximation, when certain high order mixed derivatives of the approximated function happen to be bounded in $L_{2}$. It was shown by Nitsche (2006) that this regularity constraint can be dramatically reduced by considering best $N$-term approximation from tensor product wavelet bases. When the function is the solution of some well-posed operator equation, dimension independent approximation rates can be practically realized in linear complexity by adaptive wavelet algorithms, assuming that the infinite stiffness matrix of the operator with respect to such a basis is highly compressible. Applying piecewise smooth wavelets, we verify this compressibility for general, non-separable elliptic PDEs in tensor domains. Applications of the general theory developed include adaptive Galerkin discretizations of multiple scale homogenization problems and of anisotropic equations which are robust, i.e., independent of the scale parameters, resp. of the size of the anisotropy.
\end{abstract}

\section{Motivation And BACKGround}

1.1. Adaptive wavelet algorithms. For some boundedly invertible linear operator $A: H \rightarrow H^{\prime}$, where $H$ is some separable Hilbert space with dual $H^{\prime}$, and some $f \in H^{\prime}$, let us consider the problem of finding $u \in H$ such that

$$
A u=f .
$$

Specifically, we will be interested in (systems of) elliptic PDEs in variational form. Assuming that we have a Riesz basis $\Psi=\left\{\psi_{\lambda}: \lambda \in \Lambda\right\}$ for $H$ available, e.g., a wavelet basis, formally viewed as a column vector, by writing $u=\boldsymbol{u}^{T} \Psi$ the above problem is equivalent to finding $\boldsymbol{u} \in \ell_{2}=\ell_{2}(\Lambda)$ satisfying the infinite matrix-vector system

$$
A u=f .
$$

Received by the editor May 8, 2006 and, in revised form, December 6, 2006

2000 Mathematics Subject Classification. Primary 41A25, 41A46, 41A63, 65D32, 65N12, $65 \mathrm{~T} 60$

Key words and phrases. Elliptic PDE's on (high) dimensional product domains, multiple scale homogenization, tensor product approximation, sparse grids, wavelets, best $N$-term approximation, optimal computational complexity, matrix compression.

This work was performed in part in the IHP network "Breaking Complexity" of the EC under contract HPRN-CT-2002-00286 and supported by the Netherlands Organization for Scientific Research and by the Swiss BBW under grant BBW 02.0418 .

(C)2007 American Mathematical Society Reverts to public domain 28 years from publication 
Here the stiffness matrix $\boldsymbol{A}:=\langle\Psi, A \Psi\rangle: \ell_{2} \rightarrow \ell_{2}$ is boundedly invertible and $f:=\langle\Psi, f\rangle \in \ell_{2}$, with $\langle\cdot, \cdot\rangle$ denoting the duality pairing on $H \times H^{\prime}$. We will use $\|\cdot\|$ to denote $\|\cdot\|_{\ell_{2}}$ or $\|\cdot\|_{\ell_{2} \rightarrow \ell_{2}}$.

When one is prepared to spend say $N$ arithmetical operations and storage locations, the most economical approximation for $\boldsymbol{u}$ with respect to $\|\cdot\|$ is its best $N$-term approximation $\boldsymbol{u}_{N}$, being the vector consisting of the $N$ largest coefficients of $\boldsymbol{u}$ in modulus and zeros elsewhere. Clearly, in the case that not all coefficients of $\boldsymbol{u}$ are known, a best $N$-term approximation is not practically accessible.

To relate approximation of $\boldsymbol{u}$ with that of $u$, note that for any $\boldsymbol{v} \in \ell_{2},\|\boldsymbol{u}-\boldsymbol{v}\| \bar{\sim}$ $\left\|u-\boldsymbol{v}^{T} \Psi\right\|_{H}$. Here, and in the remainder of this paper, by $C \lesssim D$ we mean that $C$ can be bounded by a multiple of $D$, independently of parameters which $C$ and $D$ may depend on (sometimes with the exception of the parameter $n$ that will be introduced in Sect. 1.2). Obviously, $C \gtrsim D$ is defined as $D \lesssim C$, and $C \approx D$ as $C \lesssim D$ and $C \gtrsim D$.

Using the fact that $\boldsymbol{u}$ is given as the solution of (1.1), when for some $s>0$ it happens to be the approximation class

$$
\mathcal{A}_{\infty}^{s}=\left\{\boldsymbol{v} \in \ell_{2}: \sup _{N} N^{s}\left\|\boldsymbol{v}-\boldsymbol{v}_{N}\right\|<\infty\right\},
$$

the adaptive wavelet algorithms from CDD01, CDD02, GHS05] are proven to produce a sequence of approximations that converge with this rate $s$, requiring a number of operations equivalent to their length, under the assumptions that one knows how to produce approximations for $\mathbf{f}$ of length $N$ in $\mathcal{O}(N)$ operations that converge with rate $s$, and that $\boldsymbol{A}$ can be sufficiently well approximated by computable sparse matrices. These assumptions are made to be able to control the cost of the successive approximate residual computations inside these iterative algorithms. More specifically, concerning $\boldsymbol{A}$, one assumes that for some $s^{*}>s$, this infinite matrix is $s^{*}$-computable, meaning that

for each $q \in \mathbb{N}:=\{0,1, \ldots\}$, an infinite matrix $\boldsymbol{A}_{q}$ can be constructed that has in each row and column $\mathcal{O}\left(2^{q}\right)$ non-zero entries, computable in $\mathcal{O}\left(2^{q}\right)$ operations, which satisfies $\left\|\boldsymbol{A}-\boldsymbol{A}_{q}\right\| \lesssim 2^{-q \bar{s}}$ for any constant $\bar{s}<s^{*}$.

Without the condition concerning the cost of computing the entries, $\boldsymbol{A}$ is called $s^{*}$-compressible. For proving $s^{*}$-computability, typically one first shows that it is $s^{*}$-compressible, where the $\boldsymbol{A}_{q}$ are constructed by dropping entries from $\boldsymbol{A}$, and then one shows that the remaining entries can be approximated with suitable quadrature, that keeps the error on level $\mathcal{O}\left(2^{-q \bar{s}}\right)$ for any $\bar{s}<s^{*}$, where the average number of operations per entry over each row and column is $\mathcal{O}(1)$. The reason to expect that $\boldsymbol{A}$ is $s^{*}$-compressible are the properties of the wavelets, that is, their vanishing moments and smoothness.

The condition on $\boldsymbol{f}$ has to be verified for the right hand side at hand. From $\boldsymbol{u} \in$ $\mathcal{A}_{\infty}^{s}$ and $\boldsymbol{A}$ being $s^{*}$-compressible with $s^{*}>s$, it follows that in any case $\boldsymbol{f} \in \mathcal{A}_{\infty}^{s}$, meaning that apart from the cost for creating them, suitable approximations for $f$ do exist.

In the above references, it is assumed that $\boldsymbol{A}$ is symmetric and positive definite. Other invertible systems can be put into this form by forming the normal equations $\boldsymbol{A}^{T} \boldsymbol{A} \boldsymbol{u}=\boldsymbol{A}^{T} \boldsymbol{f}$. As shown in Gan06, for mildly non-symmetric or indefinite equations, the algorithms from CDD01, GHS05] can be applied directly to the original system, avoiding the squaring of the condition number. 
Let us now study the value of $s$ for which $\boldsymbol{u} \in \mathcal{A}_{\infty}^{s}$ might be expected. To this end, for $\tau \in(0, \infty)$, define $\mathcal{A}_{\tau}^{s}=\left\{\boldsymbol{v} \in \ell_{2}: \sum_{N \in \mathbb{N}}\left(N^{s}\left\|\boldsymbol{v}-\boldsymbol{v}_{N}\right\|\right)^{\tau} N^{-1}<\infty\right\}$. This class is thus (slightly) smaller than $\mathcal{A}_{\infty}^{s}$. We think of $H$ as a Sobolev space of order $\ell$ on a $d$-dimensional domain, possibly incorporating essential boundary conditions. We have in mind some scalar elliptic PDE of order $2 \ell$ together with appropriate boundary conditions. Then for standard, isotropic, sufficiently smooth wavelets of order $p>\ell$, it is known (e.g., DeV98, Coh03]) that, with $\tau=\left(s+\frac{1}{2}\right)^{-1}$,

$$
u \in B_{\tau}^{s d+\ell}\left(L_{\tau}\right) \Longleftrightarrow \boldsymbol{u} \in \mathcal{A}_{\tau}^{s} \quad\left(s \in\left(0, \frac{p-\ell}{d}\right)\right),
$$

where $B_{\tau}^{s d+\ell}\left(L_{\tau}\right)$ is a certain Besov space measuring " $s d+\ell$ orders of smoothness in $L_{\tau}$ " with the secondary smoothness parameter also equal to $\tau$, possibly incorporating essential boundary conditions. The upshot of this result on non-linear approximation is that $B_{\tau}^{s d+\ell}\left(L_{\tau}\right)$ is much larger that the Sobolev space $H^{s d+\ell}$, membership of which is needed to get the same rate with standard linear approximation methods of order $d$. Since the method of approximation is of order $p$, note that by imposing whatever smoothness conditions on $u$, for $s>(p-\ell) / d, \boldsymbol{u} \in \mathcal{A}_{\infty}^{s}$ cannot be guaranteed, or actually be expected.

Thinking, as we will do, of wavelets that are piecewise smooth, and globally $C^{r}$, with $r=-1$ meaning no global smoothness conditions, the smoothness condition on the wavelets reads as $(p-\ell) / d \geq p-r-3 / 2$, i.e., $(p-\ell) / d \geq 1 / 2$ for spline wavelets where $r=p-2$.

Returning to the adaptive wavelet schemes, when the differential operator has sufficiently smooth coefficients, and the wavelets have $\tilde{p}>p-2 \ell$ vanishing moments, then, as shown in GS04, the corresponding stiffness matrix $\boldsymbol{A}$ is $s^{*}$-computable for some $s^{*}>(p-\ell) / d$, for $d>1$ additionally assuming that $\left(r+\frac{3}{2}-\ell\right) /(d-1)>$ $(p-\ell) / d$, which for $r=p-2$ reads as $(p-\ell) / d>1 / 2$. We conclude that under these conditions, for the whole relevant range of $s$, the adaptive wavelet algorithms converge with the best possible rate $s$ in linear complexity.

1.2. PDE's on (high dimensional) product domains. In terms of the number of degrees of freedom, the best possible rate of approximation $(p-\ell) / d$ decreases with increasing space dimension $d$. This effect is known as the curse of dimensionality. Moreover, simultaneously the smoothness conditions required to achieve a certain rate increase.

Fortunately, high dimensional PDEs are usually formulated on simple domains $\boldsymbol{\Omega}$, being the $n$-fold product of component domains $\Omega_{m} \subset \mathbb{R}^{d_{m}}, m=1, \ldots, n$, of dimension $d=\operatorname{dim} \boldsymbol{\Omega}=\sum_{m=1}^{n} d_{m}$. We mention only mathematical finance (pricing of derivative contracts on baskets of $n$ assets under stochastic volatility models with $d_{m}-1 \geq 0$ 'hidden' volatility drivers for the $m$ th risky asset with the case $d_{m}=1$ corresponding to deterministic volatility; see, e.g., HMS05), multiscale problems (elliptic homogenization problems with $n$ separated length scales); see, e.g., HS05, stochastic PDEs (the computation of $n$-point correlation functions for random solutions); see, e.g., vPS06.

In the non-adaptive, linear approximation setting it is known that under circumstances the curse of dimensionality can be circumvented by so called sparse tensor product approximation schemes: with $H^{s}\left(\Omega_{m}\right)$ being either the standard Sobolev space, or a closed subspace of it involving essential boundary conditions, 
for $\boldsymbol{t} \in[0, \infty)^{n}, \ell \in[0, \infty)$, let

$$
H^{\boldsymbol{t}, \ell}(\boldsymbol{\Omega}):=\bigcap_{k=1}^{n} \bigotimes_{m=1}^{n} H^{\boldsymbol{t}_{m}+\delta_{m k} \ell}\left(\Omega_{m}\right)
$$

with $\delta_{m k}$ being the Kronecker delta. So, as extreme cases, in particular $H^{\boldsymbol{t}, 0}(\boldsymbol{\Omega})=$ $\bigotimes_{m=1}^{n} H^{\boldsymbol{t}_{m}}\left(\Omega_{m}\right)$, known as a Sobolev space with dominating mixed derivatives, whereas $H^{\mathbf{0}, \ell}(\boldsymbol{\Omega})$ is (isomorphic to) the standard Sobolev space of order $\ell$ on $\boldsymbol{\Omega}$ (possibly with appropriate boundary conditions). Using suitable approximation schemes of order $p_{m}>\boldsymbol{t}_{m}+\ell$ in the $m$ th coordinate space, the (optimized) tensor product approximation with $N$ unknowns results in an error in the $H^{\boldsymbol{t}, \ell}(\boldsymbol{\Omega})$-norm of $\mathcal{O}\left(N^{-s}\right)$ with rate

$$
s=\min _{m} \frac{p_{m}-\boldsymbol{t}_{m}-\ell}{d_{m}},
$$

which is thus independent of $n$, under the assumption that certain higher order mixed derivatives of the approximated function are bounded in $L_{2}(\boldsymbol{\Omega})$; see [GK00] and references cited there.

Recently, in Nit06, corresponding results have been shown for non-linear approximation using a tensor product wavelet basis for $H^{t, \ell}(\boldsymbol{\Omega})$, reducing the regularity constraint to (nearly) its minimum: in the $m$ th coordinate direction, assume we have available a set of sufficiently smooth wavelets $\left\{\psi_{\lambda}^{(m)}: \lambda \in \Lambda_{m}\right\}$ of order $p_{m}>\boldsymbol{t}_{m}+\ell$ such that, with $|\lambda|$ denoting the level of the corresponding wavelet, for a range of $\ell^{\prime} \in \mathbb{R}$ that includes $\boldsymbol{t}_{m}$ and $\boldsymbol{t}_{m}+\ell$,

$$
\left\{2^{-|\lambda| \ell^{\prime}} \psi_{\lambda}^{(m)}: \lambda \in \Lambda_{m}\right\} \text { is a Riesz basis for } H^{\ell^{\prime}}\left(\Omega_{m}\right) .
$$

Here, since we need that, properly scaled, the wavelets generate Riesz bases for more than one Sobolev space, we made explicit the scaling that depends on the level and smoothness index $\ell^{\prime}$. This is in contrast to the previous section, where for convenience, we tacitly adapted the scaling of the wavelets to the relevant Sobolev space. As shown in [GO95, GK00, as a consequence of the scaling (1.3) we have, with $\boldsymbol{\Lambda}:=\prod_{m=1}^{n} \Lambda_{m}$ and, for any block-multiindex $\boldsymbol{\lambda} \in \boldsymbol{\Lambda}$ of length $|\boldsymbol{\lambda}|:=\left(\left|\boldsymbol{\lambda}_{1}\right|, \ldots,\left|\boldsymbol{\lambda}_{n}\right|\right) \in \mathbb{R}^{n}$

$$
\left\{2^{-\boldsymbol{t} \cdot|\boldsymbol{\lambda}|-\ell\||\boldsymbol{\lambda}|\|_{\infty}} \bigotimes_{m=1}^{n} \psi_{\boldsymbol{\lambda}_{m}}^{(m)}: \boldsymbol{\lambda} \in \boldsymbol{\Lambda}\right\} \text { is a Riesz basis for } H^{\boldsymbol{t}, \ell}(\boldsymbol{\Omega}) .
$$

Now with $\boldsymbol{u}$ denoting the representation of any $u \in H^{\boldsymbol{t}, \ell}(\boldsymbol{\Omega})$ with respect to the tensor product Riesz basis, the question is for which $s$ we might expect $\boldsymbol{u} \in \mathcal{A}_{\infty}^{s}$, and under which regularity conditions.

Suppose that the wavelets in each coordinate direction $m$ are sufficiently smooth such that for $\ell^{\prime} \in\left\{\boldsymbol{t}_{m}, \boldsymbol{t}_{m}+\ell\right\}$, with $\boldsymbol{u}_{m}$ denoting the representation of $u_{m} \in H^{\ell^{\prime}}(\Omega)$ with respect to (1.3), and $\tau=\left(s+\frac{1}{2}\right)^{-1}$,

$$
u_{m} \in B_{\tau}^{s d_{m}+\ell^{\prime}}\left(L_{\tau}\left(\Omega_{m}\right)\right) \Longleftrightarrow \boldsymbol{u}_{m} \in \mathcal{A}_{\tau}^{s} \quad\left(s \in\left(0, \frac{p_{m}-\ell^{\prime}}{d_{m}}\right)\right),
$$

which is (1.2) in the coordinate space for two cases. Then, as shown in Nit06] (the proof given there for $\Omega_{m}=(0,1)$ carries over verbatim to the more general situation under consideration here)

$$
u \in \bigcap_{k=1}^{n} \bigotimes_{m=1}^{n} B_{\tau}^{s d_{m}+\boldsymbol{t}_{m}+\delta_{m k} \ell}\left(L_{\tau}\left(\Omega_{m}\right)\right) \Longleftrightarrow \boldsymbol{u} \in \mathcal{A}_{\tau}^{s} \quad s \in\left(0, \min _{m} \frac{p_{m}-\boldsymbol{t}_{m}-\ell}{d_{m}}\right),
$$


where $\otimes_{\tau}$ denotes the so-called " $\tau$ tensor product" (we refer to Nit06 for a definition and properties of $\otimes_{\tau}$ for $\left.0<\tau<1\right)$. For piecewise smooth, globally $C^{r_{m}}\left(\Omega_{m}\right)$ wavelets in the coordinate spaces, the aforementioned smoothness conditions are satisfied when $\left(p_{m}-\boldsymbol{t}_{m}-\ell\right) / d_{m} \geq p_{m}-r_{m}-3 / 2$, i.e., when $\left(p_{m}-\boldsymbol{t}_{m}-\ell\right) / d_{m} \geq \frac{1}{2}$ for spline wavelets.

Compared to (1.2), in (1.6) not only the curse of dimensionality has been removed, but also the regularity condition needed to achieve a certain rate $s$ has been reduced. Indeed, a comparison for $\boldsymbol{t}=0$, and, for simplicity, $\ell=0, d_{m} \equiv 1$, shows that only the order of the mixed derivatives involved in the smoothness requirements from (1.6) are equal to those from (1.2); the order of the (primary) directional derivatives involved in (1.6) is independent of $n$. E.g., solutions of elliptic PDEs on polyhedra in more than 2 space dimensions generally exhibit anisotropic singularities that, using isotropic basis functions, can be approximated with limited rates only, regardless of the order of approximation. Such solutions, transported as a function on the unit cube, have arbitrary regularity in the scale of spaces at the left hand side of (1.6) (see [Nit06).

The result (1.6) deals with best $N$-term approximations, which are not practically accessible. When $u$ is given as the solution of $A u=f$ with $A: H^{t, \ell}(\boldsymbol{\Omega}) \rightarrow$ $H^{\boldsymbol{t}, \ell}(\boldsymbol{\Omega})^{\prime}$ boundedly invertible and $f \in H^{\boldsymbol{t}, \ell}(\boldsymbol{\Omega})^{\prime}$, the adaptive wavelet methods discussed in the setting of isotropic wavelet bases can be applied verbatim. To achieve the high, dimension independent rates of the best $N$-term approximations from tensorized wavelet bases shown in Nit06, the resulting infinite stiffness matrix $\boldsymbol{A}$, however, has to be $s^{*}$-computable with

$$
s^{*}>\min _{m} \frac{p_{m}-\boldsymbol{t}_{m}-\ell}{d_{m}} .
$$

E.g., thinking of $p_{m}=p$ and $\boldsymbol{t}_{m}=0$, this is a much stronger condition than is needed with isotropic wavelets of order $p$, where $s^{*}>(p-\ell) / d=(p-\ell) / \sum_{m} d_{m}$ is required.

1.3. Results from this paper. A (scalar) PDE that, in variational form, corresponds to a bounded differential operator $A: H^{\boldsymbol{t}, \ell}(\boldsymbol{\Omega}) \rightarrow H^{\boldsymbol{t}, \ell}(\boldsymbol{\Omega})^{\prime}$, has the following general form:

$$
\langle A u, v\rangle=\sum_{\left\{\boldsymbol{\alpha}, \boldsymbol{\beta}:\|\max (|\boldsymbol{\alpha}|-\boldsymbol{t}, \mathbf{0})\|_{1},\|\max (|\boldsymbol{\beta}|-\boldsymbol{t}, \mathbf{0})\|_{1} \leq \ell\right\}} \int_{\boldsymbol{\Omega}} g_{\boldsymbol{\alpha}, \boldsymbol{\beta}} \boldsymbol{\partial}^{\boldsymbol{\alpha}} v \boldsymbol{\partial}^{\boldsymbol{\beta}} u
$$

with $g_{\boldsymbol{\alpha}, \boldsymbol{\beta}} \in L_{\infty}(\boldsymbol{\Omega})$. Here for block-multiindices $\boldsymbol{\alpha} \in \prod_{m=1}^{n} \mathbb{N}^{d_{m}}$, we set $\boldsymbol{\partial}^{\boldsymbol{\alpha}}:=$ $\bigotimes_{m=1}^{n} \partial_{m}^{\boldsymbol{\alpha}_{m}}$ with $\partial_{m}^{\boldsymbol{\alpha}_{m}}$ acting in the component domain $\Omega_{m}$, and the vector of their orders $|\boldsymbol{\alpha}|:=\left(\left|\boldsymbol{\alpha}_{1}\right|, \ldots,\left|\boldsymbol{\alpha}_{n}\right|\right) \in \mathbb{R}^{n}$. For $s, \boldsymbol{s}^{\prime} \in \mathbb{R}^{n}$, we set $\max (s, s):=$ $\left(\max \left(s_{1}, s_{1}^{\prime}\right), \ldots, \max \left(s_{n}, \boldsymbol{s}_{n}^{\prime}\right)\right)$, and analogously $\min \left(\boldsymbol{s}, \boldsymbol{s}^{\prime}\right)$. Under additional conditions, e.g., coercivity, $A$ has a bounded inverse. Examples will be given in Sect. 2

An entry $\boldsymbol{A}_{\boldsymbol{\lambda}, \boldsymbol{\lambda}^{\prime}}$ of the stiffness matrix $\boldsymbol{A}=\left(\boldsymbol{A}_{\boldsymbol{\lambda}, \boldsymbol{\lambda}^{\prime}}\right)_{\boldsymbol{\lambda}, \boldsymbol{\lambda}^{\prime} \in \boldsymbol{\Lambda} \times \boldsymbol{\Lambda}}$ with respect to the tensor product wavelet basis (1.4) reads as

$$
\left.\times \sum_{\left\{\boldsymbol{\alpha}, \boldsymbol{\beta}:\|\max (|\boldsymbol{\alpha}|-\boldsymbol{t}, \mathbf{0})\|_{1},\|\max (|\boldsymbol{\beta}|-\boldsymbol{t}, \mathbf{0})\|_{1} \leq \ell\right\}} \int_{\boldsymbol{\Omega}} g_{\boldsymbol{\alpha}, \boldsymbol{\beta}} \boldsymbol{\partial}^{\boldsymbol{\alpha} \cdot\left(|\boldsymbol{\lambda}|+\left|\boldsymbol{\lambda}^{\prime}\right|\right)-\ell\left(\|{ }^{\prime}\left(\bigotimes_{m=1}^{n}\left\|_{\infty}+\right\|\left|\boldsymbol{\lambda}^{\prime}\right| \|_{\infty}\right)\right.} \psi_{\boldsymbol{\lambda}_{m}}^{(m)}\right) \boldsymbol{\partial}^{\boldsymbol{\beta}}\left(\bigotimes_{m=1}^{n} \psi_{\boldsymbol{\lambda}_{m}^{\prime}}^{(m)}\right) .
$$


We will show that when the parameters $\left(p_{m}, r_{m}, \tilde{p}_{m}\right)$ characterizing the (piecewise smooth) wavelets in the coordinate spaces $\Omega_{m}$, with $\tilde{p}_{m}$ being the number of vanishing moments, are chosen to satisfy

$$
\begin{aligned}
\min _{\left\{\boldsymbol{\alpha}, \boldsymbol{\beta}:\|\max (\boldsymbol{\alpha}-\boldsymbol{t}, \mathbf{0})\|_{1},\|\max (\boldsymbol{\beta}-\boldsymbol{t}, \mathbf{0})\|_{1} \leq \ell\right\}}\left[\min _{m} \frac{\tilde{p}_{m}+\min \left(\left|\boldsymbol{\alpha}_{m}\right|,\left|\boldsymbol{\beta}_{m}\right|\right)}{d_{m}},\right. \\
\left.\min _{\left\{m: d_{m}>1\right\}} \frac{r_{m}+\frac{3}{2}-\max \left(\left|\boldsymbol{\alpha}_{m}\right|,\left|\boldsymbol{\beta}_{m}\right|\right)}{d_{m}-1}\right]>\min _{m} \frac{p_{m}-\boldsymbol{t}_{m}-\ell}{d_{m}},
\end{aligned}
$$

the (mixed) derivatives of sufficiently high order of the coefficients $g_{\boldsymbol{\alpha}, \boldsymbol{\beta}}$ are in $L_{\infty}(\boldsymbol{\Omega})$, and the PDEs in the coordinate spaces are appended with either periodic or homogeneous Dirichlet boundary conditions, then, with $s^{*}$ as in (1.7), $\boldsymbol{A}$ is $s^{*}$ computable. With this the adaptive wavelet methods for solving systems $A u=$ $f$ are shown to converge with the optimal rate in linear complexity. Note that the constant involved in the error bound for the approximations produced by the adaptive wavelet algorithm might depend on $n$.

Remark 1.1. The condition (1.9) is in any case satisfied when $\tilde{p}_{m}>p_{m}-\boldsymbol{t}_{m}-\ell$ and, when $d_{m}>1, \frac{r_{m}+\frac{3}{2}-\boldsymbol{t}_{m}-\ell}{d_{m}-1}>\frac{p_{m}-\boldsymbol{t}_{m}-\ell}{d_{m}}$, i.e., for spline wavelets, $\frac{p_{m}-\boldsymbol{t}_{m}-\ell}{d_{m}}>\frac{1}{2}$.

To arrive at this result on computability of the stiffness matrix, we show that under these conditions, for any $\boldsymbol{\alpha}, \boldsymbol{\beta}$ in the sum from (1.8), the matrix $\boldsymbol{I}^{(\boldsymbol{\alpha}, \boldsymbol{\beta})}=$ $\left(\boldsymbol{I}_{\boldsymbol{\lambda}, \boldsymbol{\lambda}^{\prime}}^{(\boldsymbol{\alpha}, \boldsymbol{\beta})}\right)_{\boldsymbol{\lambda}, \boldsymbol{\lambda}^{\prime} \in \boldsymbol{\Lambda} \times \boldsymbol{\Lambda}}$ defined by

$$
\boldsymbol{I}_{\boldsymbol{\lambda}, \boldsymbol{\lambda}^{\prime}}^{(\boldsymbol{\alpha}, \boldsymbol{\beta})}:=2^{-|\boldsymbol{\lambda}| \cdot|\boldsymbol{\alpha}|} 2^{-\left|\boldsymbol{\lambda}^{\prime}\right| \cdot|\boldsymbol{\beta}|} \int_{\boldsymbol{\Omega}} g_{\boldsymbol{\alpha}, \boldsymbol{\beta}} \boldsymbol{\partial}^{\boldsymbol{\alpha}}\left(\bigotimes_{m=1}^{n} \psi_{\boldsymbol{\lambda}_{m}}^{(m)}\right) \cdot \boldsymbol{\partial}^{\boldsymbol{\beta}}\left(\bigotimes_{m=1}^{n} \psi_{\boldsymbol{\lambda}_{m}^{\prime}}^{(m)}\right)
$$

is $s^{*}$-computable (Theorem 6.2), so that from

$$
2^{-\boldsymbol{t} \cdot\left(|\boldsymbol{\lambda}|+\left|\boldsymbol{\lambda}^{\prime}\right|\right)-\ell\left(\||\boldsymbol{\lambda}|\|_{\infty}+\|\| \boldsymbol{\lambda}^{\prime} \mid \|_{\infty}\right)} \leq 2^{-\left(|\boldsymbol{\lambda}| \cdot|\boldsymbol{\alpha}|+\left|\boldsymbol{\lambda}^{\prime}\right| \cdot|\boldsymbol{\beta}|\right)}
$$

for such $\boldsymbol{\alpha}, \boldsymbol{\beta}$, the result follows.

For separable PDEs, i.e., when each $g_{\boldsymbol{\alpha}, \boldsymbol{\beta}}$ is a (finite sum of) product(s) of functions on the coordinate spaces, $\boldsymbol{A}$ is a finite sum of tensor products of stiffness matrices resulting from PDEs in the coordinate spaces, each of them being $s^{*}$ compressible. As shown in [Nit06], as a consequence, $\boldsymbol{A}$ is $s^{*}$-compressible. In this paper, we extend this result to non-separable PDEs, i.e., to coefficients $g_{\boldsymbol{\alpha}, \boldsymbol{\beta}}$ that are not necessarily of the aforementioned special form.

Remark 1.2. Although we consider in this paper only elliptic PDEs, our results on $s^{*}$-compressibility and $s^{*}$-computability of the stiffness matrix also have immediate applications to the efficient solution of parabolic problems by implicit timestepping procedures as discussed, e.g., in vPS04.

Remark 1.3. Generally, (1.11) is not sharp, resulting in compression and quadrature rules that quantitatively can be improved. In order not to (further) complicate the exposition, we did not make an attempt to do so. For the highest order terms, i.e., those with $\||\boldsymbol{\alpha}|\|_{1}=\||\boldsymbol{\beta}|\|_{1}=\|\boldsymbol{t}\|_{1}+\ell$, equality holds if and only if $|\boldsymbol{\lambda}|_{m} \equiv\|\boldsymbol{\lambda}\|_{\infty}$ and $\left|\boldsymbol{\lambda}^{\prime}\right|_{m} \equiv\left\|\boldsymbol{\lambda}^{\prime}\right\|_{\infty}$. For lower order terms, if present, (1.11) is always crude, resulting in conditions on the number of vanishing moments that are unnecessarily strong. For practical wavelet constructions, however, that usually yield $\tilde{p}_{m} \geq p_{m}$, this does not do any harm. 
The remaining part of this paper is organized as follows. In Sect. 2, we give three examples of applications, the last two involving some extensions of the setting we discussed so far. In Sect. 3, we describe our assumptions on the wavelets in the coordinate spaces, and give some basic estimates. In Sect. 4. we show $s^{*}$ compressibility of the $\boldsymbol{I}^{(\boldsymbol{\alpha}, \boldsymbol{\beta})}$. After discussing sparse quadrature rules in Sect. 5 , in Sect. 6, we show that the $\boldsymbol{I}^{(\boldsymbol{\alpha}, \boldsymbol{\beta})}$ are $s^{*}$-computable. We summarize our results in Sect. 7.

\section{Applications}

2.1. Diffusion problems. In elliptic and parabolic diffusion problems arising, e.g., in mathematical finance, differential equation of interest is the Dirichlet problem

$$
-\operatorname{div} G(x) \nabla u=-\sum_{m, m^{\prime}=1}^{n} \partial_{m} g_{m, m^{\prime}}(x) \partial_{m^{\prime}} u=f \quad \text { in } \quad \boldsymbol{\Omega}, \quad u=0 \quad \text { on } \quad \partial \boldsymbol{\Omega}
$$

where $\Omega_{m}=(0,1)$, i.e., $d_{m}=1$, and so $\boldsymbol{\Omega}=(0,1)^{n}$, and where the diffusion coefficient $G(x) \in \mathbb{R}_{s y m}^{n \times n}$ is assumed to have smooth component functions $g_{m, m^{\prime}}(x)$ and to be uniformly positive definite. The corresponding bilinear form

$$
a(u, v)=\sum_{m, m^{\prime}=1}^{n} \int_{\Omega} g_{m, m^{\prime}}(x) \partial_{m} u \partial_{m^{\prime}} v
$$

defines a boundedly invertible operator $H_{0}^{\mathbf{0}, 1}(\boldsymbol{\Omega}) \rightarrow H_{0}^{\mathbf{0}, 1}(\boldsymbol{\Omega})^{\prime}$, where the subscript 0 refers to the incorporation of the homogeneous Dirichlet boundary conditions in the definition of the Sobolev space.

Remark 2.1. The diffusion operator (2.1) is (part of) the infinitesimal generator of geometric Brownian Motion which appears in mathematical finance. Other generators $A$ appear in connection with other Markovian processes; they may be degenerate (see, e.g., [HMS05] and, for the Riesz basis property in this case, [BSS04]), but always fit into our abstract tensor framework.

2.2. Elliptic $n$-scale homogenization. In All92, AB96, the following class of scalar elliptic periodic homogenization problems with $n \geq 2$ scales was considered: let $D \subset \mathbb{R}^{d_{1}}$ be a bounded domain and let $Y_{2}, \ldots, Y_{n} \subset \mathbb{R}^{\bar{d}_{1}}$ be fundamental periods or "cells". Further, let

$$
B\left(x, y_{2}, \ldots, y_{n}\right) \in L^{\infty}\left(D \times Y_{2} \times \ldots \times Y_{n} ; \mathbb{R}_{s y m}^{d_{1} \times d_{1}}\right)
$$

be a matrix function depending on $n$ variables taking values in the space $\mathbb{R}_{s y m}^{d_{1} \times d_{1}}$ of symmetric matrices of size $d_{1} \times d_{1} ; B$ is assumed periodic with respect to $y_{m}$ with period $Y_{m}=[0,1]^{d_{1}}$ for each $m=2, \ldots, n$ (different cells $Y_{m}$ of possibly different dimensions on each scale would not cause any difficulties). We assume that $B$ is bounded and uniformly positive definite, i.e., there is a constant $\gamma>0$ such that for all $\xi \in \mathbb{R}^{d_{1}}$

$$
\gamma|\xi|^{2} \leq \xi^{\top} B\left(x, y_{2}, \ldots, y_{n}\right) \xi \leq \gamma^{-1}|\xi|^{2},
$$

uniformly in the "slow variable" $x \in D$ and in the fast variables $y_{m} \in Y_{m}, m=$ $2, \ldots, n$.

For a scale parameter $\varepsilon>0$, we consider the Dirichlet problem

$$
-\operatorname{div} B^{\varepsilon} \nabla u^{\varepsilon}=f \quad \text { in } D, \quad u^{\varepsilon}=0 \text { on } \partial D .
$$


The $d_{1} \times d_{1}$ matrix $B^{\varepsilon}$ is assumed to depend on $\varepsilon$ with multiple scales in the following sense: There are $n-1$ positive scale functions $\varepsilon_{1}(\varepsilon)>\varepsilon_{2}(\varepsilon)>\ldots>\varepsilon_{n-1}(\varepsilon)$ of $\varepsilon$ that converge to 0 monotonically when $\varepsilon \rightarrow 0$ and that are scale separated:

$$
\lim _{\varepsilon \rightarrow 0} \varepsilon_{m+1} / \varepsilon_{m}=0, \quad m=1, \ldots, n-2,
$$

so that for all $x \in D$ it holds that

$$
B^{\varepsilon}(x)=B\left(x, \frac{x}{\varepsilon_{1}}, \ldots, \frac{x}{\varepsilon_{n-1}}\right) .
$$

When $n=2$, the scale separation assumption (2.4) is void and we have the classical two-scale homogenization problem

$$
-\operatorname{div} B\left(x, \frac{x}{\varepsilon}\right) \nabla u^{\varepsilon}=f
$$

which is dealt with thoroughly in the book by Bensoussan, Lions and Papanicolaou BLP78. The purpose of homogenization is the study of the limit of $u^{\varepsilon}$ when $\varepsilon$ converges to 0 and to get an asymptotic expansion of $u^{\varepsilon}$ to infer its behaviour when $\varepsilon>0$ is small.

It was shown in All92, AB96 that for small, positive $\varepsilon>0$ the homogenization limit and all oscillations of the solution $u^{\varepsilon}$ can be described to leading order by a high-dimensional, $\varepsilon$-independent limit problem, the so-called $n$-scale limit problem. It is posed on the tensor domain $\boldsymbol{\Omega}=D \times Y_{2} \times \ldots \times Y_{n}$, which is a special case of our general setting with $d_{m}=d_{1}$ for all $m$ and thus of total dimension $d=n d_{1}$. For notational convenience, in the following we write $Y_{1}$ instead of $D$, and $y_{1}$ instead of $x$.

For the variational formulation of the $n$-scale limit, with $\boldsymbol{t}^{(m)}:=(0, \ldots, 0,1) \in$ $\mathbb{N}^{m}, m=1, \ldots, n$, we define the space

$$
\mathbf{V}=\prod_{m=1}^{n} H^{\boldsymbol{t}^{(m)}, 0}\left(Y_{1} \times \ldots \times Y_{m}\right)
$$

and endow it with the natural product norm

$$
\left\|\left\{\phi_{m}\right\}\right\|\left\|^{2}=\sum_{m=1}^{n}\right\| \phi_{m} \|_{H^{t^{(m)}, 0}\left(Y_{1} \times \ldots \times Y_{m}\right)}^{2} .
$$

Here, following the definitions given in Sect. 1.2,

$H^{\boldsymbol{t}^{(m)}, 0}\left(Y_{1} \times \ldots \times Y_{m}\right)=\left(\bigotimes_{k=1}^{m-1} L_{2}\left(Y_{k}\right)\right) \bigotimes \tilde{H}^{1}\left(Y_{m}\right) \sim L^{2}\left(Y_{1} \times \ldots \times Y_{m-1} ; \tilde{H}^{1}\left(Y_{m}\right)\right)$

with $\tilde{H}^{1}\left(Y_{1}\right)=H_{0}^{1}\left(Y_{1}\right)$, and for $m>1, \tilde{H}^{1}\left(Y_{m}\right)=H_{\#}^{1}\left(Y_{m}\right)$ being the subspace of $H^{1}\left(Y_{m}\right)$ of functions whose 1-periodic extension to $\mathbb{R}^{d_{1}}$ belongs to $H_{\text {loc }}^{1}\left(\mathbb{R}^{d_{1}}\right)$ and whose mean over $Y_{m}$ vanishes.

Note that $\mathbf{V}$ is the product of function spaces over tensor product domains of dimension $d_{1}, 2 d_{2}, \ldots, n d_{1}$.

Proposition 2.2 ([Al192, AB96]). The bilinear form

$$
a\left(\left\{u_{m}\right\} ;\left\{\phi_{m^{\prime}}\right\}\right)=\int_{Y_{1} \times \ldots \times Y_{n}} B\left(y_{1}, \ldots, y_{n}\right)\left(\sum_{m=1}^{n} \nabla_{y_{m}} u_{m}\right) \cdot\left(\sum_{m^{\prime}=1}^{n} \nabla_{y_{m^{\prime}}} \phi_{m^{\prime}}\right) d y_{1} \ldots d y_{n}
$$


is bounded and coercive on $\mathbf{V} \times \mathbf{V}$. For given $f \in L_{2}\left(Y_{1}\right)$, with $U=\left(u_{1}, \ldots, u_{n}\right)$ denoting the unique solution of the variational problem: find $\left\{u_{m}\right\} \in \mathbf{V}$ such that

$$
a\left(\left\{u_{m}\right\} ;\left\{\phi_{m^{\prime}}\right\}\right)=\int_{Y_{1}} f \phi d y_{1}, \quad \forall\left\{\phi_{m^{\prime}}\right\} \in \mathbf{V},
$$

the solution $u^{\varepsilon}$ of the problem (2.3) converges weakly to $u_{1}$ in $H_{0}^{1}(\Omega)$ and the gradient $\nabla u^{\varepsilon} n$-scale converges to $\sum_{m=1}^{n} \nabla_{y_{m}} u_{m}$.

For a proof and the definition of $n$-scale convergence, we refer to AB96, Th. 2.11, eq. (2.9), and Def. 2.3]. Note that the problem (2.8) is independent of $\varepsilon$, and can therefore be solved numerically and approximately at a robust, i.e., $\varepsilon$-independent convergence rate.

We are interested in solving (2.8) by adaptive wavelet methods. Therefore, below we transfer results announced in Sect. 1.3 for scalar equations, to the system (2.8) of equations. To this end, for $m=1, \ldots, n$ we let $\left\{\psi_{\lambda}^{(m)}: \lambda \in \Lambda_{m}\right\}$ be a collection of wavelets of order $p$, such that for $\ell^{\prime}=0$ or $\ell^{\prime}=1,\left\{2^{-|\lambda| \ell^{\prime}} \psi_{\lambda}^{(m)}: \lambda \in \Lambda_{m}\right\}$ is a Riesz basis for $L_{2}\left(Y_{m}\right)$ or $\tilde{H}^{1}\left(Y_{m}\right)$, respectively, and, with $\boldsymbol{u}_{m}$ denoting the representation of $u_{m} \in L_{2}\left(Y_{m}\right)$ or $u_{m} \in \tilde{H}^{1}\left(Y_{m}\right)$, respectively, such that for $s \in\left(0, \frac{p-\ell^{\prime}}{d_{1}}\right)$ with $\tau=\left(s+\frac{1}{2}\right)^{-1}, u_{m} \in B_{\tau}^{s d_{1}+\ell^{\prime}}\left(L_{\tau}\left(Y_{m}\right)\right)$ if and only if $\boldsymbol{u}_{m} \in \mathcal{A}_{\tau}^{s}$, i.e., (1.5). Then

$$
\left\{2^{-\left|\boldsymbol{\lambda}_{m}\right|} \bigotimes_{k=1}^{m} \psi_{\boldsymbol{\lambda}_{k}}^{(k)}: \boldsymbol{\lambda} \in \prod_{k=1}^{m} \Lambda_{k}\right\} \text { is a Riesz basis for } H^{\left(\boldsymbol{t}^{(m)}, 0\right)}\left(\prod_{k=1}^{m} Y_{k}\right),
$$

and so the Cartesian product for $m=1, \ldots, n$ of these bases is a Riesz basis for V. With $\boldsymbol{U}=\left(\boldsymbol{u}_{1}, \ldots, \boldsymbol{u}_{n}\right)$ denoting the representation of $U=\left(u_{1}, \ldots, u_{n}\right)$ with respect to this basis, one easily verifies that $\boldsymbol{U} \in \mathcal{A}^{s}$ if and only if $\boldsymbol{u}_{m} \in \mathcal{A}^{s}$ $(1 \leq m \leq n)$. This, in turn, as we have seen in (1.6), holds for $s \in\left(0, \frac{p-1}{d_{1}}\right)$ if and only if

$$
u_{m} \in\left(\bigotimes_{k=1}^{m-1} B_{\tau}^{s d_{1}}\left(L_{\tau}\left(Y_{k}\right)\right)\right) \bigotimes_{\tau} B_{\tau}^{s d_{1}+1}\left(L_{\tau}\left(Y_{m}\right)\right)
$$

Moreover, $\boldsymbol{U} \in \mathcal{A}^{s}$ for $s>\frac{p-1}{d_{1}}$ cannot be expected by imposing whatever smoothness conditions.

To show that the adaptive wavelet methods for solving (2.8) realize the same convergence rates as that of the best $N$-term approximations, we have to show that the stiffness matrix corresponding to the bilinear form $a$ with respect to the basis of $\mathbf{V}$ is $s^{*}$-computable for some $s^{*}>\frac{p-1}{d_{1}}$. This stiffness matrix has a natural $n \times n$ block partitioning with the $\left(m, m^{\prime}\right)$ th block being the stiffness matrix corresponding to the bilinear form

$$
\sum_{\left\{\alpha \in \mathbb{N}^{d}:|\alpha|=1\right\}} \int_{Y_{1} \times \ldots \times Y_{n}} B_{m m^{\prime}} \partial_{m}^{\alpha} u_{m} \partial_{m^{\prime}}^{\alpha} \phi_{m^{\prime}} d y_{1} \ldots d y_{n}
$$

with respect to the bases for $H^{t^{(m)}, 0}\left(\prod_{k=1}^{m} Y_{k}\right)$ and $H^{t^{\left(m^{\prime}\right)}, 0}\left(\prod_{k=1}^{m^{\prime}} Y_{k}\right)$, respectively. In the case of $m \leq m^{\prime}$, this matrix is the sum over $|\alpha|=1$ of matrices 
$[\cdots]_{\boldsymbol{\lambda} \in \prod_{k=1}^{m} \Lambda_{k}, \boldsymbol{\lambda}^{\prime} \in \prod_{k=1}^{m^{\prime}} \Lambda_{k}}$, with the $\left(\boldsymbol{\lambda}, \boldsymbol{\lambda}^{\prime}\right)$ th entry given by $2^{-\left(\left|\boldsymbol{\lambda}_{m}\right|+\left|\boldsymbol{\lambda}_{m^{\prime}}^{\prime}\right|\right)}$ times

$$
\begin{gathered}
\int_{\prod_{k=1}^{m^{\prime} Y_{k}}} B_{m m^{\prime}}\left(\left(\bigotimes_{k=1}^{m-1} \psi_{\boldsymbol{\lambda}_{k}}^{(k)}\right) \otimes \partial_{m}^{\alpha} \psi_{\boldsymbol{\lambda}_{m}}^{(m)}\right) \\
\cdot\left(\left(\bigotimes_{k=1}^{m^{\prime}-1} \psi_{\boldsymbol{\lambda}_{k}^{\prime}}^{(k)}\right) \otimes \partial_{m^{\prime}}^{\alpha} \psi_{\boldsymbol{\lambda}_{m}^{\prime}}^{\left(m^{\prime}\right)}\right) d y_{1} \ldots d y_{m^{\prime}}
\end{gathered}
$$

Setting $\boldsymbol{\alpha}:=(0, \ldots, \alpha, \ldots, 0) \in \mathbb{N}^{d_{1} m^{\prime}}$, i.e., $\alpha$ on the position of the $m$ th coordinate direction, and $\boldsymbol{\beta}:=(0, \ldots, \alpha) \in \mathbb{N}^{d_{1} m^{\prime}}$, without the mean value is zero condition of functions in $H_{\#}^{1}\left(Y_{m}\right)$, the last matrix would be a submatrix of $\boldsymbol{I}^{(\boldsymbol{\alpha}, \boldsymbol{\beta})}=$ $\left(\boldsymbol{I}_{\boldsymbol{\lambda}, \boldsymbol{\lambda}^{\prime}}^{(\boldsymbol{\alpha}, \boldsymbol{\beta})}\right)_{\boldsymbol{\lambda}, \boldsymbol{\lambda}^{\prime} \in\left(\prod_{k^{\prime}=1}^{m} \Lambda_{k^{\prime}}\right)^{2}}$ from (1.10), with $g_{\boldsymbol{\alpha}, \boldsymbol{\beta}}$ reading as $B_{m m^{\prime}}$, by leaving all entries with $\left(\boldsymbol{\lambda}_{m+1}, \ldots, \boldsymbol{\lambda}_{m^{\prime}}\right) \neq 0$. With wavelets in the coordinate spaces that are piecewise smooth, globally $C^{r}$, and that have $\tilde{p}$ vanishing moments, and for a $B_{m m^{\prime}}$ that is sufficiently smooth, in Theorem 6.2 it will be shown that this $\boldsymbol{I}^{(\boldsymbol{\alpha}, \boldsymbol{\beta})}$ is $s^{*}$ computable with $s^{*}=\frac{\tilde{p}+1}{d_{1}}$ when $d_{1}=1$, and $s^{*}=\min \left(\frac{\tilde{p}+1}{d_{1}}, \frac{r+\frac{1}{2}}{d_{1}-1}\right)$ otherwise. Since a submatrix of an $s^{*}$-computable matrix is $s^{*}$-computable, we conclude that for $\tilde{p}>p-1$ and, when $d_{1}>1, \frac{r+\frac{1}{2}}{d_{1}-1}>\frac{p-1}{d_{1}}$, i.e., $\frac{p-1}{d_{1}}>\frac{1}{2}$ for spline wavelets, the adaptive wavelet methods for solving (2.8) converge with the best possible rate $s$ in linear complexity. More importantly, however, at small, positive $\varepsilon>0$ the physical solution $u^{\varepsilon}$ can be approximated in $H^{1}(D)$ in terms of $u_{1}, u_{2}, \ldots, u_{n}$ : as was shown in [AB96, Theorem 2.14, it holds (under the assumption of $H^{2}$-regularity of all components $u_{i}$ ) that, as $\varepsilon \rightarrow 0$,

$$
u^{\varepsilon}(x)-\left[u_{1}(x)+\sum_{m=2}^{n} \varepsilon_{m-1} u_{m}\left(x, \frac{x}{\varepsilon_{1}}, \ldots, \frac{x}{\varepsilon_{m-1}}\right)\right] \rightarrow 0 \quad \text { strongly in } H^{1}(D) .
$$

2.3. Anisotropic problems. We consider the model anisotropic problem

$$
-\sum_{m=1}^{n} \partial_{m} c_{m} g_{m}(x) \partial_{m} u+c_{0} u=f \quad \text { in } \quad \boldsymbol{\Omega}, \quad u=0 \quad \text { on } \quad \partial \boldsymbol{\Omega}
$$

where $\Omega_{m}=(0,1)$, i.e., $d_{m}=1$, and so $\boldsymbol{\Omega}=(0,1)^{n}, g_{m}, 1 / g_{m} \in L_{\infty}(\boldsymbol{\Omega})$ and sufficiently smooth, and the constants $c_{0} \geq 0, c_{m}>0(m=1, \ldots, n)$. Here the interest is to derive results that are valid uniformly in $\boldsymbol{c}=\left(c_{0}, \ldots, c_{m}\right)$.

The corresponding bilinear form

$$
a_{\boldsymbol{c}}(u, v)=\sum_{m=1}^{n} \int_{\Omega} c_{m} g_{m}(x) \partial_{m} u \partial_{m} v+\int_{\Omega} c_{0} g_{0}(x) u v
$$

defines a uniformly bounded invertible operator from

$$
V_{\boldsymbol{c}}:=\bigcap_{k=1}^{n} \bigotimes_{m=1}^{n} c_{m} H_{0}^{\delta_{m k}}(0,1) \cap c_{0} L_{2}(\boldsymbol{\Omega})
$$

to its dual, where $H_{0}^{0}(0,1)=L_{2}(0,1)$, and where $c H$ denotes the space $H$ equipped with the scaled norm $c^{\frac{1}{2}}\|\cdot\|_{H}$. Assuming that for $m=1, \ldots, n$, and for $\ell^{\prime}=0$ or 
$\ell^{\prime}=1,\left\{2^{-|\lambda| \ell^{\prime}} \psi_{\lambda}^{(m)}: \lambda \in \Lambda_{m}\right\}$ is a Riesz basis for $L_{2}(0,1)$ or $H_{0}^{1}(0,1)$, respectively,

$$
\left\{\left[c_{0}+\sum_{m=1}^{n} c_{m} 4^{\left|\boldsymbol{\lambda}_{m}\right|}\right]^{-\frac{1}{2}} \bigotimes_{m=1}^{n} \psi_{\boldsymbol{\lambda}_{m}}^{(m)}: \boldsymbol{\lambda} \in \boldsymbol{\Lambda}\right\} \text { is a uniform Riesz basis for } V_{\boldsymbol{c}}
$$

For a proof, we refer to GO95.

As a consequence, the resulting stiffness matrix $\boldsymbol{A}_{\boldsymbol{c}}$ defines a uniformly boundedly invertible mapping $\ell_{2} \rightarrow \ell_{2}$. Its $\left(\boldsymbol{\lambda}, \boldsymbol{\lambda}^{\prime}\right)$ th entry is given by

$$
\left[c_{0}+\sum_{m=1}^{n} c_{m} 4^{\left|\boldsymbol{\lambda}_{m}\right|}\right]^{-\frac{1}{2}}\left[c_{0}+\sum_{m=1}^{n} c_{m} 4^{\left|\boldsymbol{\lambda}_{m}^{\prime}\right|}\right]^{-\frac{1}{2}} a_{\boldsymbol{c}}\left(\bigotimes_{m=1}^{n} \psi_{\boldsymbol{\lambda}_{m}}^{(m)}, \bigotimes_{m=1}^{n} \psi_{\boldsymbol{\lambda}_{m}^{\prime}}^{(m)}\right) .
$$

Since $\left[c_{0}+\sum_{m=1}^{n} c_{m} 4^{\left|\boldsymbol{\lambda}_{m}\right|}\right]^{-\frac{1}{2}}\left[c_{0}+\sum_{m=1}^{n} c_{m} 4^{\left|\boldsymbol{\lambda}_{m}^{\prime}\right|}\right]^{-\frac{1}{2}} c_{0} \leq 1$, and, for $m=1, \ldots, n$, $\left[c_{0}+\sum_{m=1}^{n} c_{m} 4^{\left|\boldsymbol{\lambda}_{m}\right|}\right]^{-\frac{1}{2}}\left[c_{0}+\sum_{m=1}^{n} c_{m} 4^{\left|\boldsymbol{\lambda}_{m}^{\prime}\right|}\right]^{-\frac{1}{2}} c_{m} \leq 2^{-\left|\boldsymbol{\lambda}_{m}\right|} 2^{-\left|\boldsymbol{\lambda}_{m}^{\prime}\right|}$, the forthcoming Theorem 6.2, dealing with computability of $\boldsymbol{I}^{(\boldsymbol{\alpha}, \boldsymbol{\beta})}$, shows that with wavelets in the coordinate spaces of order $p_{m} \equiv p$, with $\tilde{p}_{m} \equiv \tilde{p}>p-1$ vanishing moments, $\boldsymbol{A}_{\boldsymbol{c}}$ is uniformly $s^{*}$-computable with $s^{*}=\tilde{p}>p-1$, the latter being the maximum rate of convergence that can be expected for best $N$-term approximations. So whenever for some $0<s<s^{*}$, the representation $\boldsymbol{u}$ of the solution $u$ is in $\mathcal{A}^{s}$, then given a tolerance $\varepsilon>0$, the adaptive wavelet algorithms produce approximations to $\boldsymbol{u}$ within this tolerance taking $\mathcal{O}\left(\varepsilon^{-1 / s}\left[\sup _{N} N^{s}\left\|\boldsymbol{u}-\boldsymbol{u}_{N}\right\|\right]^{1 / s}\right)$ operations uniformly in $c$.

Finally, membership of $\boldsymbol{u} \in \mathcal{A}^{s}$ is implied by that of $\boldsymbol{u} \in \mathcal{A}_{\tau}^{s}$, which for $s \in$ $(0, p-1)$ and $\tau=\left(\frac{1}{2}+s\right)^{-1}$ is equivalent, uniformly in $c$, to membership of $u \in$ $\bigcap_{k=1}^{n} \bigotimes_{m=1}^{n} c_{m} B_{\tau}^{s+\delta_{m k}}\left(L_{\tau}(0,1)\right) \cap c_{0} \bigotimes_{m=1}^{n} B_{\tau}^{s}\left(L_{\tau}(0,1)\right)$, as follows from a generalization of (1.6).

\section{WAVELETS AND BASIC ESTIMATES IN THE COORDinAte SPACES}

For $1 \leq m \leq n$, let $\Omega_{m}$ be a domain in $\mathbb{R}^{d_{m}}$. Let $\left\{\psi_{\lambda}^{(m)}: \lambda \in \Lambda_{m}\right\} \subset L_{2}\left(\Omega_{m}\right)$ be a collection of functions (wavelets), such that for some $\tilde{p}_{m} \in \mathbb{N}$,

$$
\left|\int_{\Omega_{m}} u \psi_{\lambda}^{(m)}\right| \lesssim 2^{-|\lambda|\left(\frac{d_{m}}{2}+s\right)}\|u\|_{W_{\infty}^{s}\left(\operatorname{supp} \psi_{\lambda}^{(m)}\right)}, \quad s \in\left[0, \tilde{p}_{m}\right]
$$

where $|\lambda| \in \mathbb{N}$ denotes the level of $\psi_{\lambda}^{(m)}$. The estimate 3.1) can be shown when $\left\|\psi_{\lambda}^{(m)}\right\|_{L_{2}\left(\Omega_{m}\right)} \lesssim 1$, the wavelets are locally supported, in the sense that $\operatorname{vol}\left(\operatorname{supp} \psi_{\lambda}^{(m)}\right)$ $\lesssim 2^{-|\lambda| d_{m}}$, and when if $\tilde{p}_{m}>0$, possibly after some smooth transformation of coordinates, $\psi_{\lambda}^{(m)}$ for $|\lambda|>0$ has $\tilde{p}_{m}$ vanishing moments, meaning that $\psi_{\lambda}^{(m)} \perp_{L_{2}\left(\Omega_{m}\right)}$ $P_{\tilde{p}_{m}-1}$. Actually, when $d_{m}>1$, with some constructions $\operatorname{supp} \psi_{\lambda}^{(m)}$ in (3.1) should read as a neighbourhood of $\operatorname{supp} \psi_{\lambda}^{(m)}$ with a comparable diameter. For convenience we ignore this fact, but our results extend trivially to this situation.

In addition to being locally supported, we assume that the wavelets are piecewise smooth in the following sense: For all $l \in \mathbb{N}$, there exists a collection $\left\{\Omega_{m}^{(l, v)}\right.$ : $\left.v \in \mathcal{O}_{l}^{(m)}\right\}$ of disjoint, uniformly shaped regular, open subdomains, such that $\bar{\Omega}_{m}=\bigcup_{v \in \mathcal{O}_{l}^{(m)}} \bar{\Omega}_{m}^{(l, v)}, \bar{\Omega}_{m}^{(l, v)}$ is the union of some $\bar{\Omega}_{m}^{(l+1, \tilde{v})}, \operatorname{diam}\left(\Omega_{m}^{(l, v)}\right) \approx 2^{-l}$, $\operatorname{supp} \psi_{\lambda}^{(m)}$ is connected and is the union of a uniformly bounded number of $\bar{\Omega}_{m}^{(|\lambda|, v)}$, and each $\bar{\Omega}_{m}^{(l, v)}$ has a non-empty intersection with the supports of a uniformly 
bounded number of $\psi_{\lambda}^{(m)}$ with $|\lambda|=l$. So, in particular, the singular support of $\psi_{\lambda}^{(m)}$ is part of the boundaries of the $\Omega_{m}^{(|\lambda|, v)}$.

Finally, for some $r_{m} \in \mathbb{N} \cup\{-1\}$, we will assume that

$$
\left\|\psi_{\lambda}^{(m)}\right\|_{W_{\infty}^{s}\left(\Omega_{m}\right)} \lesssim 2^{|\lambda|\left(\frac{d_{m}}{2}+s\right)}, \quad s \in\left[0, r_{m}+1\right]
$$

and that for any $s \geq 0$,

$$
\left\|\psi_{\lambda}^{(m)}\right\|_{W_{\infty}^{s}\left(\Omega_{m}^{(|\lambda|, v)}\right)} \lesssim 2^{|\lambda|\left(\frac{d_{m}}{2}+s\right)}
$$

These estimates follow from a homogeneity argument assuming that the wavelets are globally in $C^{r_{m}}\left(\Omega_{m}\right)$ which, for $r_{m}=-1$, is to be understood as possibly discontinuous wavelet functions.

In the periodic setting that was discussed in Subsect. 2.2 for the coordinate spaces $Y_{i}$ for $i>0$, the above conditions on the wavelets should be read as to hold for their periodic extensions. In a non-periodic setting, in the following Lemma 3.1 we will additionally assume homogeneous Dirichlet boundary conditions that are satisfied in the applications discussed in Sect. 2.

For convenience, in the following we restrict ourselves to the common situation that

$$
\tilde{p}_{m} \geq r_{m}+2 \text {. }
$$

With this we have presented all conditions on the wavelet we will need in our investigation of computability of stiffness matrices of PDEs with respect to tensor product wavelets. To use wavelets as a suitable discretization tool, however, more properties are required. Basically, for having (1.3) and (1.5), one needs that the wavelets have some order $p_{m}$, meaning that locally any polynomial of degree $p_{m}-1$ can be reproduced by the wavelet basis, that the wavelets generate a Riesz basis for $L_{2}\left(\Omega_{m}\right)$, and that the dual wavelets, which have order $\tilde{p}_{m}$, have some smoothness, i.e., satisfy a Bernstein inequality like (3.2). For a detailed discussion, we refer to Coh03. In the literature, one can find various constructions of suitable wavelets on the interval (e.g., DKU99, Pri06]), or cubes, and, usually via some domain decomposition approach, on general domains (e.g., DS99a, CTU99, DS99b, DS99c, HS04, Ste04]). From the introduction, recall that the larger the orders $p_{m}$ are, the better compressible the stiffness matrix should be, which will induce stronger conditions on the values of the $\tilde{p}_{m}$ and $r_{m}$.

For $\left(\lambda, \lambda^{\prime}\right) \in \Lambda_{m} \times \Lambda_{m}$, we define the indicator $i_{m}\left(\lambda, \lambda^{\prime}\right) \in\{0,1\}$ by

$$
i_{m}\left(\lambda, \lambda^{\prime}\right):=0 \text { when }\left\{\begin{array}{l}
\psi_{\lambda^{\prime}}^{(m)} \text { vanishes on } \operatorname{singsupp} \psi_{\lambda}^{(m)} \text { when }\left|\lambda^{\prime}\right| \geq|\lambda| \text {, or } \\
\psi_{\lambda}^{(m)} \text { vanishes on singsupp } \psi_{\lambda^{\prime}}^{(m)} \text { when }\left|\lambda^{\prime}\right|<|\lambda|,
\end{array}\right.
$$

and $i_{m}\left(\lambda, \lambda^{\prime}\right):=1$ otherwise; see Figure1, For $\alpha \in \mathbb{N}^{d_{m}}$, let $\partial_{m}^{\alpha}$ denote the canonical partial differentiation operator on $\Omega_{m}$ of order $|\alpha|=\sum_{k=1}^{d_{m}} \alpha_{k}$.

Lemma 3.1. For some, sufficiently smooth, real valued function $g_{m}$ on $\Omega_{m}, \lambda, \lambda^{\prime} \in$ $\Lambda_{m}$ with $|\lambda| \leq\left|\lambda^{\prime}\right|$, and $|\alpha|,|\beta| \leq r_{m}+1$, for

$$
I_{\lambda, \lambda^{\prime}, m}^{(\alpha, \beta)}:=2^{-|\lambda||\alpha|} 2^{-\left|\lambda^{\prime}\right||\beta|} \int_{\Omega_{m}} g_{m} \partial_{m}^{\alpha} \psi_{\lambda}^{(m)} \partial_{m}^{\beta} \psi_{\lambda^{\prime}}^{(m)},
$$




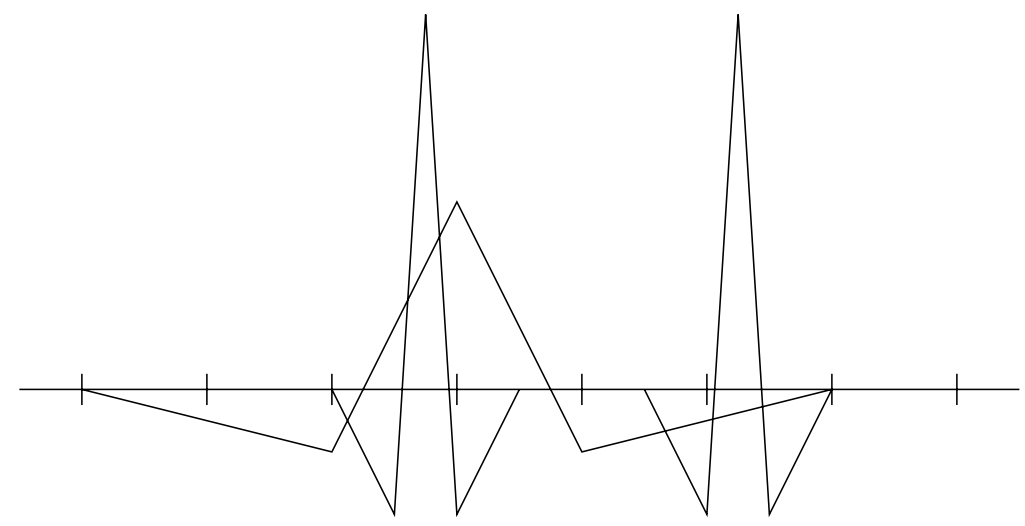

FIGURE 1. Wavelets $\psi_{\lambda^{\prime}}^{(m)}$ that vanish (right case), i.e., $i_{m}\left(\lambda, \lambda^{\prime}\right)=$ 0 , or do not vanish (left case), i.e., $i_{m}\left(\lambda, \lambda^{\prime}\right)=1$, on the singular support of a wavelet $\psi_{\lambda}^{(m)}$ with $|\lambda| \leq\left|\lambda^{\prime}\right|$. The subdivision of $\Omega_{m}$ into $\Omega_{m}^{|\lambda|, v}$ is also indicated

we have

$$
\left|I_{\lambda, \lambda^{\prime}, m}^{(\alpha, \beta)}\right| \lesssim\left\{\begin{array}{r}
2^{\left(|\lambda|-\left|\lambda^{\prime}\right|\right)\left(\frac{d_{m}}{2}+r_{m}+1-|\alpha|\right)}\left\|g_{m}\right\|_{W_{\infty}^{r_{m}+1-|\alpha|}\left(\operatorname{supp} \psi_{\lambda}^{(m)} \cap \operatorname{supp} \psi_{\lambda^{\prime}}^{(m)}\right)} \\
\text { for any } \lambda, \lambda^{\prime} \in \Lambda_{m}, \\
2^{\left(|\lambda|-\left|\lambda^{\prime}\right|\right)\left(\frac{d_{m}}{2}+\tilde{p}_{m}+|\beta|\right)}\left\|g_{m}\right\|_{W_{\infty}^{\tilde{p} m}+|\beta|}\left(\operatorname{supp} \psi_{\lambda}^{(m)} \cap \operatorname{supp} \psi_{\lambda^{\prime}}^{(m)}\right) \\
\text { when } i_{m}\left(\lambda, \lambda^{\prime}\right)=0 .
\end{array}\right.
$$

For this to hold in a non-periodic setting, we will assume that all wavelets satisfy homogeneous Dirichlet boundary conditions $\partial^{\eta} \psi_{\lambda^{\prime}}^{(m)}=0$ on $\partial \Omega_{m}$ for all $\eta \leq \beta$ with $\eta \neq \beta$.

Proof. To prove the first estimate, we distinguish between two cases. When $|\beta+\alpha| \geq r_{m}+1$, select $\gamma \leq \beta$ with $|\gamma+\alpha|=r_{m}+1$. Integration by parts, where we use the homogeneous Dirichlet boundary conditions in the non-periodic setting, and (3.2) for both $\psi_{\lambda}^{(m)}$ and $\psi_{\lambda^{\prime}}^{(m)}$ show that

$$
\begin{aligned}
\left|I_{\lambda, \lambda^{\prime}, m}^{(\alpha, \beta)}\right|= & 2^{-|\lambda||\alpha|} 2^{-\left|\lambda^{\prime}\right||\beta|}\left|\int_{\operatorname{supp} \psi_{\lambda}^{(m)} \cap \operatorname{supp} \psi_{\lambda^{\prime}}^{(m)}}(-1)^{|\gamma|} \partial_{m}^{\gamma}\left(g_{m} \partial_{m}^{\alpha} \psi_{\lambda}^{(m)}\right) \partial_{m}^{\beta-\gamma} \psi_{\lambda^{\prime}}^{(m)}\right| \\
& \lesssim 2^{-|\lambda||\alpha|} 2^{-\left|\lambda^{\prime}\right||\beta|}\left\|g_{m}\right\|_{W_{\infty}^{r_{m}+1-|\alpha|}\left(\operatorname{supp} \psi_{\lambda}^{(m)} \cap \operatorname{supp} \psi_{\lambda^{\prime}}^{(m)}\right)} \\
\times 2^{-\left|\lambda^{\prime}\right| d_{m}} 2^{|\lambda|\left(\frac{d_{m}}{2}+r_{m}+1\right)} 2^{\left|\lambda^{\prime}\right|\left(\frac{d_{m}}{2}+|\beta|-r_{m}+1+|\alpha|\right)} & \\
= & \left\|g_{m}\right\|_{W_{\infty}^{r_{m}+1-|\alpha|}\left(\operatorname{supp} \psi_{\lambda}^{(m)} \cap \operatorname{supp} \psi_{\lambda^{\prime}}^{(m)}\right)} 2^{\left(|\lambda|-\left|\lambda^{\prime}\right|\right)\left(\frac{d_{m}}{2}+r_{m}+1-|\alpha|\right)}
\end{aligned}
$$


When $|\beta+\alpha| \leq r_{m}+1$, by integration by parts, (3.2), and (3.1) with $s+|\alpha|+|\beta|=$ $r_{m}+1$, where we used that $r_{m}+1-|\alpha|-|\beta| \leq r_{m}+1 \leq \tilde{p}_{m}-1 \leq \tilde{p}_{m}$, we have

$$
\begin{aligned}
\left|I_{\lambda, \lambda^{\prime}, m}^{(\alpha, \beta)}\right|= & 2^{-|\lambda||\alpha|} 2^{-\left|\lambda^{\prime}\right||\beta|}\left|\int_{\Omega_{m}}(-1)^{|\beta|} \partial_{m}^{\beta}\left(g_{m} \partial_{m}^{\alpha} \psi_{\lambda}^{(m)}\right) \psi_{\lambda^{\prime}}^{(m)}\right| \\
\lesssim & 2^{-|\lambda||\alpha|} 2^{-\left|\lambda^{\prime}\right||\beta|}\left\|g_{m}\right\|_{W_{\infty}^{r_{m}+1-|\alpha|}\left(\operatorname{supp} \psi_{\lambda}^{(m)} \cap \operatorname{supp} \psi_{\lambda^{\prime}}^{(m)}\right)} \\
& \times 2^{|\lambda|\left(\frac{d_{m}}{2}+r_{m}+1\right)} 2^{-\left|\lambda^{\prime}\right|\left(\frac{d_{m}}{2}+r_{m}+1-|\alpha|-|\beta|\right)} \\
= & \left\|g_{m}\right\|_{W_{\infty}^{r_{m}+1-|\alpha|}\left(\operatorname{supp} \psi_{\lambda}^{(m)} \cap \operatorname{supp} \psi_{\lambda^{\prime}}^{(m)}\right)} 2^{\left(|\lambda|-\left|\lambda^{\prime}\right|\right)\left(\frac{d_{m}}{2}+r_{m}+1-|\alpha|\right)} .
\end{aligned}
$$

When $\psi_{\lambda^{\prime}}^{(m)}$ vanishes on singsupp $\psi_{\lambda}^{(m)}$, from (3.1) and (3.3) we have

$$
\begin{aligned}
\left|I_{\lambda, \lambda^{\prime}, m}^{(\alpha, \beta)}\right|= & 2^{-|\lambda||\alpha|} 2^{-\left|\lambda^{\prime}\right||\beta|}\left|\int_{\Omega_{m}}(-1)^{|\beta|} \partial_{m}^{\beta}\left(g_{m} \partial_{m}^{\alpha} \psi_{\lambda}^{(m)}\right) \psi_{\lambda^{\prime}}^{(m)}\right| \\
\lesssim & 2^{-|\lambda||\alpha|} 2^{-\left|\lambda^{\prime}\right||\beta|}\left\|g_{m}\right\|_{W_{\infty}^{|\beta|+\tilde{p}_{m}}\left(\operatorname{supp} \psi_{\lambda}^{(m)} \cap \operatorname{supp} \psi_{\lambda^{\prime}}^{(m)}\right)} \\
& \times 2^{|\lambda|\left(\frac{d_{m}}{2}+|\alpha|+|\beta|+\tilde{p}_{m}\right)} 2^{-\left|\lambda^{\prime}\right|\left(\frac{d_{m}}{2}+\tilde{p}_{m}\right)} \\
= & 2^{\left(|\lambda|-\left|\lambda^{\prime}\right|\right)\left(\frac{d_{m}}{2}+\tilde{p}_{m}+|\beta|\right)}\left\|g_{m}\right\|_{W_{\infty}^{\tilde{p}_{m}+|\beta|}\left(\operatorname{supp} \psi_{\lambda}^{(m)} \cap \operatorname{supp} \psi_{\lambda^{\prime}}^{(m)}\right)},
\end{aligned}
$$

which is the second estimate.

All our results concerning compressibility and computability of differential operators with respect to tensorized wavelet bases will be based on this Lemma 3.1. Unfortunately, in order to get optimal results, we cannot rely solely on the first estimate, but instead, we have to use that for the vast majority of pairs $\left(\lambda, \lambda^{\prime}\right) \in$ $\Lambda_{m} \times \Lambda_{m}$ the stronger second estimate is valid.

\section{Compressibility}

We will use the following notations, some of them already introduced in Sect.1.2 $\boldsymbol{\Omega}:=\prod_{m=1}^{n} \Omega_{m}, \tilde{\boldsymbol{p}}:=\left(\tilde{p}_{1}, \ldots, \tilde{p}_{m}\right), \boldsymbol{r}:=\left(r_{1}, \ldots, r_{n}\right), \mathbf{1}:=(1, \ldots, 1), \boldsymbol{d}:=$ $\left(d_{1}, \ldots, d_{n}\right)$, and $\boldsymbol{\Lambda}:=\prod_{m=1}^{n} \Lambda_{m}$. For $\boldsymbol{\alpha} \in \prod_{m=1}^{n} \mathbb{N}^{d_{m}}$, we set $\boldsymbol{\partial}^{\boldsymbol{\alpha}}:=\bigotimes_{m=1}^{n} \partial_{m}^{\boldsymbol{\alpha}_{m}}$, and $|\boldsymbol{\alpha}|:=\left(\left|\boldsymbol{\alpha}_{1}\right|, \ldots,\left|\boldsymbol{\alpha}_{n}\right|\right) \in \mathbb{R}^{n}$. For $\boldsymbol{\lambda} \in \boldsymbol{\Lambda}$, we set $|\boldsymbol{\lambda}|:=\left(\left|\boldsymbol{\lambda}_{1}\right|, \ldots,\left|\boldsymbol{\lambda}_{n}\right|\right) \in \mathbb{R}^{n}$. For $s, s^{\prime} \in \mathbb{R}^{n}$, we set $\min \left(s, s^{\prime}\right):=\left(\min \left(s_{1}, s_{1}^{\prime}\right), \ldots, \min \left(s_{n}, s_{n}^{\prime}\right)\right)$, analogously $\max \left(s, s^{\prime}\right)$, and $\left|s-s^{\prime}\right|=\left(\left|s_{1}-s_{1}^{\prime}\right|, \ldots,\left|s_{n}-s_{n}^{\prime}\right|\right)$. For $\left(\boldsymbol{\lambda}, \boldsymbol{\lambda}^{\prime}\right) \in \boldsymbol{\Lambda} \times \boldsymbol{\Lambda}$, we set

$$
\boldsymbol{i}\left(\boldsymbol{\lambda}, \boldsymbol{\lambda}^{\prime}\right)=\left(i_{1}\left(\boldsymbol{\lambda}_{1}, \boldsymbol{\lambda}_{1}^{\prime}\right), \ldots, i_{n}\left(\boldsymbol{\lambda}_{n}, \boldsymbol{\lambda}_{n}^{\prime}\right)\right) \in\{0,1\}^{n}
$$

inducing a decomposition of $\boldsymbol{\Lambda} \times \boldsymbol{\Lambda}$ into $2^{n}$ disjoint sets.

Theorem 4.1. Let $\boldsymbol{\alpha}, \boldsymbol{\beta} \in \prod_{m=1}^{n} \mathbb{N}^{d_{m}}$ with $\max (|\boldsymbol{\alpha}|,|\boldsymbol{\beta}|) \leq \boldsymbol{r}+\mathbf{1}$. For $g$ being a real-valued function on $\boldsymbol{\Omega}$ with, for $\gamma \in \prod_{m=1}^{n} \mathbb{N}^{d_{m}}$,

$$
\partial^{\gamma} g \in L_{\infty}(\boldsymbol{\Omega}), \quad|\gamma| \leq \tilde{\boldsymbol{p}}+\min (|\boldsymbol{\alpha}|,|\boldsymbol{\beta}|),
$$

let the infinite matrix $\boldsymbol{I}^{(\boldsymbol{\alpha}, \boldsymbol{\beta})}=\left(\boldsymbol{I}_{\boldsymbol{\lambda}, \boldsymbol{\lambda}^{\prime}}^{(\boldsymbol{\alpha}, \boldsymbol{\beta})}\right)_{\boldsymbol{\lambda}, \boldsymbol{\lambda}^{\prime} \in \boldsymbol{\Lambda}}$ be defined by

$$
\boldsymbol{I}_{\boldsymbol{\lambda}, \boldsymbol{\lambda}^{\prime}}^{(\boldsymbol{\alpha}, \boldsymbol{\beta})}:=2^{-|\boldsymbol{\lambda}| \cdot|\boldsymbol{\alpha}|} 2^{-\left|\boldsymbol{\lambda}^{\prime}\right| \cdot|\boldsymbol{\beta}|} \int_{\boldsymbol{\Omega}} g \cdot \boldsymbol{\partial}^{\boldsymbol{\alpha}} \bigotimes_{m=1}^{n} \psi_{\boldsymbol{\lambda}_{m}}^{(m)} \cdot \boldsymbol{\partial}^{\boldsymbol{\beta}} \bigotimes_{m=1}^{n} \psi_{\boldsymbol{\lambda}_{m}^{\prime}}^{(m)} .
$$

Defining, for $\boldsymbol{i} \in\{0,1\}^{n}, \boldsymbol{z}^{(\boldsymbol{i})}=\left(z_{1}^{\left(\boldsymbol{i}_{1}\right)}, \ldots, z_{n}^{\left(\boldsymbol{i}_{n}\right)}\right) \in \mathbb{R}^{n}$ by

$$
z_{m}^{(i)}= \begin{cases}\tilde{p}_{m}+\min \left(\left|\boldsymbol{\alpha}_{m}\right|,\left|\boldsymbol{\beta}_{m}\right|\right) & \text { when } i=0, \\ r_{m}+\frac{3}{2}-\max \left(\left|\boldsymbol{\alpha}_{m}\right|,\left|\boldsymbol{\beta}_{m}\right|\right) & \text { when } i=1,\end{cases}
$$


for any $q \in \mathbb{N}$ we define the compressed matrix $\boldsymbol{I}_{q}^{(\boldsymbol{\alpha}, \boldsymbol{\beta})}$ as follows: we drop the entry $\boldsymbol{I}_{\boldsymbol{\lambda}, \boldsymbol{\lambda}^{\prime}}^{(\boldsymbol{\alpha}, \boldsymbol{\beta})}$ when

$$
|| \boldsymbol{\lambda}^{\prime}|-| \boldsymbol{\lambda}|| \cdot \boldsymbol{z}^{\left(\boldsymbol{i}\left(\boldsymbol{\lambda}, \boldsymbol{\lambda}^{\prime}\right)\right)}>q .
$$

Then the resulting error in the matrix can be bounded by

$$
\left\|\boldsymbol{I}^{(\boldsymbol{\alpha}, \boldsymbol{\beta})}-\boldsymbol{I}_{q}^{(\boldsymbol{\alpha}, \boldsymbol{\beta})}\right\| \lesssim q^{n-1} 2^{-q},
$$

and, with

$$
s^{*}:=\left[\max _{1 \leq m \leq n} \max \left(\frac{d_{m}}{\tilde{p}_{m}+\min \left(\left|\boldsymbol{\alpha}_{m}\right|,\left|\boldsymbol{\beta}_{m}\right|\right)}, \frac{d_{m}-1}{r_{m}+\frac{3}{2}-\max \left(\left|\boldsymbol{\alpha}_{m}\right|,\left|\boldsymbol{\beta}_{m}\right|\right)}\right)\right]^{-1},
$$

the number of non-zero entries per row and column of $\boldsymbol{I}_{q}^{(\boldsymbol{\alpha}, \boldsymbol{\beta})}$ can be bounded on some absolute multiple of

$$
q^{n-1} 2^{q / s^{*}}
$$

in particular showing that $\boldsymbol{I}^{(\boldsymbol{\alpha}, \boldsymbol{\beta})}$ is $s^{*}$-compressible.

Note that by $\tilde{p}_{m} \geq r_{m}+2$, the more coordinate directions $m$ there are in which the interior of the support of one of $\psi_{\boldsymbol{\lambda}_{m}}^{(m)}$ or $\psi_{\boldsymbol{\lambda}_{m}^{\prime}}^{(m)}$ has non-empty intersection with the singular support of the other, the more stringent is the dropping criterion. Furthermore, note that with the definition of (4.2), Lemma 3.1 can be reformulated as

$$
\begin{aligned}
& \left|I_{\lambda, \lambda^{\prime}, m}^{(\alpha, \beta)}\right| \lesssim 2^{-|| \lambda|-| \lambda^{\prime}||\left(\frac{d_{m}}{2}+z_{m}^{\left(i_{m}\left(\lambda, \lambda^{\prime}\right)\right)}-\frac{i_{m}\left(\lambda, \lambda^{\prime}\right)}{2}\right)}
\end{aligned}
$$

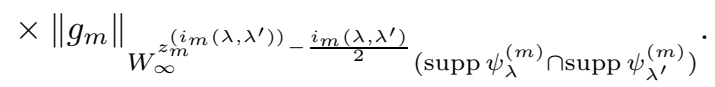

To prove Theorem 4.1 we start with a lemma.

Lemma 4.2. For any $q \in \mathbb{N}$, let $\boldsymbol{B}_{q}=\left(\left(\boldsymbol{B}_{q}\right)_{\boldsymbol{\lambda}, \boldsymbol{\lambda}^{\prime}}\right)_{\boldsymbol{\lambda}, \boldsymbol{\lambda}^{\prime} \in \boldsymbol{\Lambda}}$ be a matrix, partitioned into blocks $\left(\boldsymbol{B}_{q}\right)_{\boldsymbol{l}, \boldsymbol{l}^{\prime}}=\left(\left(\boldsymbol{B}_{q}\right)_{\boldsymbol{\lambda}, \boldsymbol{\lambda}^{\prime}}\right)_{|\boldsymbol{\lambda}|=\boldsymbol{l},\left|\boldsymbol{\lambda}^{\prime}\right|=\boldsymbol{l}^{\prime}}$, such that for some fixed $\boldsymbol{w} \in(0, \infty)^{n}$ and $\bar{s}>0$, the maximal number of non-zero entries in each row of $\left(\boldsymbol{B}_{q}\right)_{\boldsymbol{l}, \boldsymbol{l}^{\prime}}$ or column of $\left(\boldsymbol{B}_{q}\right)_{\boldsymbol{l}^{\prime}, \boldsymbol{l}}$ is bounded by some absolute multiple of

$$
2^{\max \left(\boldsymbol{l}^{\prime}-\boldsymbol{l}, 0\right) \cdot \boldsymbol{w} / \bar{s}}
$$

and

$$
\left(\boldsymbol{B}_{q}\right)_{\boldsymbol{l}, \boldsymbol{l}^{\prime}}=0 \text { when }\left|\boldsymbol{l}^{\prime}-\boldsymbol{l}\right| \cdot \boldsymbol{w}>q .
$$

Then the number of non-zero entries in each row and column of $\boldsymbol{B}_{q}$ is for $q \rightarrow \infty$ bounded by $\mathcal{O}\left(q^{n-1} 2^{q / \bar{s}}\right)$.

Proof. It is sufficient to count the number of non-zero entries $\left(\boldsymbol{B}_{q}\right)_{\boldsymbol{\lambda}, \boldsymbol{\lambda}^{\prime}}$ for any fixed $\boldsymbol{\lambda}$ and all $\boldsymbol{\lambda}^{\prime}$ with $\left|\boldsymbol{\lambda}^{\prime}\right| \geq|\boldsymbol{\lambda}|$, which can be bounded by some absolute multiple of

$$
\sum_{\left\{\boldsymbol{l}^{\prime}: 0 \leq\left(\boldsymbol{l}^{\prime}-|\boldsymbol{\lambda}|\right) \cdot \boldsymbol{w} \leq q\right\}} 2^{\left(\boldsymbol{l}^{\prime}-|\boldsymbol{\lambda}|\right) \cdot \boldsymbol{w} / \bar{s}} \lesssim q^{n-1} 2^{q / \bar{s}} .
$$

Proof of Theorem 4.1. Suppressing the dependence on $(\boldsymbol{\alpha}, \boldsymbol{\beta})$ in the notation, we write $\boldsymbol{I}=\sum_{\boldsymbol{i} \in\{0,1\}^{n}} \boldsymbol{I}^{(\boldsymbol{i})}\left(\boldsymbol{I}_{q}=\sum_{\boldsymbol{i} \in\{0,1\}^{n}} \boldsymbol{I}_{q}^{(\boldsymbol{i})}\right)$, where $\boldsymbol{I}^{(\boldsymbol{i})}\left(\boldsymbol{I}_{q}^{(\boldsymbol{i})}\right)$ contains all nonzero entries with indices $\boldsymbol{\lambda}, \boldsymbol{\lambda}^{\prime}$ with $\boldsymbol{i}\left(\boldsymbol{\lambda}, \boldsymbol{\lambda}^{\prime}\right)=\boldsymbol{i}$. By the dropping rule (4.3), the nonzero part of $\boldsymbol{I}_{q}^{(\boldsymbol{i})}$ consists of those blocks $\boldsymbol{I}_{\boldsymbol{l}, \boldsymbol{l}^{\prime}}^{(\boldsymbol{i})}=\left(\boldsymbol{I}_{\boldsymbol{\lambda}, \boldsymbol{\lambda}^{\prime}}^{(\boldsymbol{i})}\right)|\boldsymbol{\lambda}|=\boldsymbol{l},\left|\boldsymbol{\lambda}^{\prime}\right|=\boldsymbol{l}^{\prime}$ with $\left|\boldsymbol{l}^{\prime}-\boldsymbol{l}\right| \cdot \boldsymbol{z}^{(\boldsymbol{i})} \leq q$. 
By the local supports and the piecewise smoothness of the wavelets, the number of non-zero entries in each row of $\boldsymbol{I}_{\boldsymbol{l}, \boldsymbol{l}^{\prime}}^{(\boldsymbol{i})}$ or column of $\boldsymbol{I}_{\boldsymbol{l}^{\prime}, \boldsymbol{l}}^{(\boldsymbol{i})}$ can be bounded on some absolute multiple of

$$
2^{\max \left(\boldsymbol{l}^{\prime}-\boldsymbol{l}, 0\right) \cdot(\boldsymbol{d}-\boldsymbol{i})} .
$$

By definition, $s^{*}$ is the largest constant such that $\boldsymbol{d}-\boldsymbol{i} \leq \boldsymbol{z}^{(\boldsymbol{i})} / s^{*}$. By an application of Lemma 4.2, we conclude that the number of non-zero entries in each row or column of $\boldsymbol{I}_{q}^{(\boldsymbol{i})}$ is $\mathcal{O}\left(q^{n-1} 2^{q / s^{*}}\right)$.

By (4.5) and the assumption (4.1), a tensor product argument (e.g., [LC85]) shows that

$$
\begin{aligned}
\left|\boldsymbol{I}_{\boldsymbol{\lambda}, \boldsymbol{\lambda}^{\prime}}^{(\boldsymbol{\alpha}, \boldsymbol{\beta})}\right| \lesssim & 2^{-|| \boldsymbol{\lambda}^{\prime}|-| \boldsymbol{\lambda}|| \cdot\left(\frac{d}{2}+\boldsymbol{z}^{\left(i\left(\boldsymbol{\lambda}, \boldsymbol{\lambda}^{\prime}\right)\right)}-\frac{\boldsymbol{i ( \boldsymbol { \lambda } , \boldsymbol { \lambda } ^ { \prime } )}}{2}\right)} \\
& \times \max _{|\boldsymbol{\gamma}| \leq \boldsymbol{z}^{\left(i\left(\boldsymbol{\lambda}, \boldsymbol{\lambda}^{\prime}\right)\right)}-\frac{i\left(\boldsymbol{\lambda}, \boldsymbol{\lambda}^{\prime}\right)}{2}}\left\|\boldsymbol{\partial}^{\boldsymbol{\gamma}} g\right\|_{L^{\infty}\left(\prod_{m=1}^{n} \operatorname{supp} \psi_{\boldsymbol{\lambda} m}^{(m)} \cap \operatorname{supp} \psi_{\boldsymbol{\lambda}_{m}^{\prime}}^{(m)}\right)^{\cdot}}
\end{aligned}
$$

By bounding $\left\|\boldsymbol{I}_{\boldsymbol{l}, \boldsymbol{l}^{\prime}}^{(\boldsymbol{i})}\right\|^{2}$ on the product of its maximal absolute row-sum and its maximal absolute column-sum (Schur lemma), we find that

$$
\left\|\boldsymbol{I}_{\boldsymbol{l}, \boldsymbol{l}^{\prime}}^{(\boldsymbol{i})}\right\| \lesssim 2^{-\left|\boldsymbol{l}^{\prime}-\boldsymbol{l}\right| \cdot \boldsymbol{z}^{(i)}}
$$

From $\left\|\boldsymbol{I}^{(\boldsymbol{i})}-\boldsymbol{I}_{q}^{(\boldsymbol{i})}\right\|^{2} \leq \max _{\boldsymbol{l}} \sum_{\left\{\boldsymbol{l}^{\prime}:\left|\boldsymbol{l}^{\prime}-\boldsymbol{l}\right| \cdot \boldsymbol{z}^{(i)}>q\right\}}\left\|\boldsymbol{I}_{\boldsymbol{l}, \boldsymbol{l}^{\prime}}^{(\boldsymbol{i})}\right\| \times \max _{\boldsymbol{l}^{\prime}} \sum_{\left\{l:\left|\boldsymbol{l}^{\prime}-\boldsymbol{l}\right| \cdot \boldsymbol{z}^{(i)}>q\right\}}\left\|\boldsymbol{I}_{\boldsymbol{l}, \boldsymbol{l}^{\prime}}^{(\boldsymbol{i})}\right\|$, we conclude that

$$
\left\|\boldsymbol{I}^{(\boldsymbol{i})}-\boldsymbol{I}_{q}^{(\boldsymbol{i})}\right\| \lesssim q^{n-1} 2^{-q}
$$

which completes the proof.

When, say for $\left|\boldsymbol{\lambda}_{m}^{\prime}\right| \geq\left|\boldsymbol{\lambda}_{m}\right|, i_{m}\left(\boldsymbol{\lambda}_{m}, \boldsymbol{\lambda}_{m}^{\prime}\right)=1$, i.e., $\operatorname{supp} \psi_{\boldsymbol{\lambda}_{m}}^{(m)} \cap \operatorname{sing} \operatorname{supp} \psi_{\boldsymbol{\lambda}_{m}^{\prime}}^{(m)} \neq \emptyset$, in the above proof we had to apply the less favourable first bound from Lemma 3.1 . On the other hand, since $\operatorname{sing} \operatorname{supp} \psi_{\boldsymbol{\lambda}_{m}^{\prime}}^{(m)}$ is only $\left(d_{m}-1\right)$-dimensional, the larger $\sum_{m=1}^{n} \boldsymbol{i}_{m}$ is, the more sparse the block $\boldsymbol{I}_{\boldsymbol{l}, \boldsymbol{l}^{\prime}}^{(\boldsymbol{i})}$ is, meaning that we could keep more of these blocks and still respect the complexity bound (this explains the factor $d_{m}-1$ instead of $d_{m}$ in the expression $\frac{d_{m}-1}{r_{m}+\frac{3}{2}-\max \left(\left|\boldsymbol{\alpha}_{m}\right|,\left|\boldsymbol{\beta}_{m}\right|\right)}$ from (4.4)). This better sparsity also resulted in a sharper estimate for $\left\|\boldsymbol{I}_{\boldsymbol{l}, \boldsymbol{l}^{\prime}}^{(\boldsymbol{i})}\right\|$ in terms of an upper bound for its entries using the Schur lemma (this explains the factor $r_{m}+\frac{3}{2}$ instead of $r_{m}+1$ in the expression $\frac{d_{m}-1}{r_{m}+\frac{3}{2}-\max \left(\left|\boldsymbol{\alpha}_{m}\right|,\left|\boldsymbol{\beta}_{m}\right|\right)}$, as well as in the definition of $z_{m}^{(i)}$ in (4.2) $)$.

\section{Quadrature}

We study the problem of approximating the (remaining) entries $\boldsymbol{I}_{\boldsymbol{\lambda}, \boldsymbol{\lambda}^{\prime}}^{(\boldsymbol{\alpha}, \boldsymbol{\beta})}$ with numerical quadrature. We assume that for $1 \leq m \leq n$ and $a_{m}, b_{m} \geq 0$, to approximate $I_{\lambda, \lambda^{\prime}, m}^{(\alpha, \beta)}\left(\lambda, \lambda^{\prime} \in \Lambda_{m}\right)$ from (3.4), where $g_{m}(y):=g\left(x_{1}, \ldots, x_{m-1}, y, x_{m+1}, \ldots, x_{n}\right)$ with fixed $x \in \boldsymbol{\Omega}$, we have available a family of quadrature rules $\left(Q_{\lambda, \lambda^{\prime}, m, N}^{(\alpha, \beta)}\right)_{N \in \mathbb{N}}$, where $Q_{\lambda, \lambda^{\prime}, m, N}^{(\alpha, \beta)}$ has $\mathcal{O}(N)$ abscissae, such that for some $k_{m} \in \mathbb{N}$,

$$
\begin{aligned}
& \left|I_{\lambda, \lambda^{\prime}, m}^{(\alpha, \beta)}-Q_{\lambda, \lambda^{\prime}, m, N}^{(\alpha, \beta)}\right| \\
& \quad \lesssim N^{-a_{m}} 2^{-|| \lambda|-| \lambda^{\prime}||\left(\frac{d_{m}}{2}+b_{m}\right)}\left\|g_{m}\right\|_{W_{\infty}^{k_{m}}\left(\operatorname{supp} \psi_{\lambda}^{(m)} \cap \operatorname{supp} \psi_{\lambda^{\prime}}^{(m)}\right)} .
\end{aligned}
$$


Before continuing, we verify this assumption in a common situation. Let us assume that $\left|\lambda^{\prime}\right| \geq|\lambda|$. Suppose that for any $l^{\prime} \in \mathbb{N}$ and $v^{\prime} \in \mathcal{O}_{l^{\prime}}^{(m)}$, there exists a sufficiently smooth transformation of coordinates $\kappa_{m}$, with derivatives bounded uniformly in $l^{\prime}$ and $v^{\prime}$, such that for some $e_{m} \in \mathbb{N}$, and all $\left|\lambda^{\prime}\right|=l^{\prime}$,

$$
\left.\psi_{\lambda^{\prime}}^{(m)} \circ \kappa\right|_{\kappa_{m}^{-1}\left(\Omega_{m}^{\left(l^{\prime}, v^{\prime}\right)}\right)} \in P_{e_{m}-1} .
$$

In the following, without loss of generality, for notational convenience, we take $\kappa_{m}=\mathrm{id}$.

To approximate an integral $\int_{\Omega_{m}^{\left(l^{\prime}, v^{\prime}\right)}} f$, for any $k \in \mathbb{N}$ we assume to have available composite quadrature rules $Q_{\Omega_{m}^{\left(l^{\prime}, v^{\prime}\right), N}}(f)$ of fixed order (i.e, the degree of polynomial exactness plus one) $k$, and variable $\operatorname{rank} N$. The rank $N$ of a composite quadrature formula denotes the number of subdomains on which the elementary quadrature formula is applied. Since the order $k$ of $Q_{\Omega_{m}^{\left(l^{\prime}, v^{\prime}\right)}, N}$ is fixed, the number of abscissae in the composite rule $Q_{\Omega_{m}^{\left(l^{\prime}, v^{\prime}\right), N}}$ is $\mathcal{O}(N)$. We assume that the composite quadrature rule $Q_{\Omega_{m}^{\left(l^{\prime}, v^{\prime}\right), N}}$ satisfies the error estimate

$$
\begin{aligned}
& \quad\left|\int_{\Omega_{m}^{\left(l^{\prime}, v^{\prime}\right)}} f-Q_{\Omega_{m}^{\left(l^{\prime}, v^{\prime}\right)}, N}(f)\right| \\
& \quad \lesssim \operatorname{vol}\left(\Omega_{m}^{\left(l^{\prime}, v^{\prime}\right)}\right) N^{-k / d_{m}} \operatorname{diam}\left(\Omega_{m}^{\left(l^{\prime}, v^{\prime}\right)}\right)^{k}\|f\|_{W_{\infty}^{k}\left(\Omega_{m}^{\left(l^{\prime}, v^{\prime}\right)}\right)}
\end{aligned}
$$

(cf. GS04, §2]).

To find an upper bound for the quadrature error when these rules are applied with integrand $2^{-|\lambda|\left|\boldsymbol{\alpha}_{m}\right|} 2^{-\left|\lambda^{\prime}\right|\left|\boldsymbol{\beta}_{m}\right|} g_{m} \partial_{m}^{\boldsymbol{\alpha}_{m}} \psi_{\lambda}^{(m)} \partial_{m}^{\boldsymbol{\beta}_{m}} \psi_{\lambda^{\prime}}^{(m)}$, we have to bound $\left(\partial_{m}^{\rho} g_{m}\right)\left(\partial_{m}^{\sigma} \partial_{m}^{\boldsymbol{\alpha}_{m}} \psi_{\lambda}^{(m)}\right)\left(\partial_{m}^{\tau} \partial_{m}^{\boldsymbol{\beta}_{m}} \psi_{\lambda^{\prime}}^{(m)}\right)$ for $|\rho+\sigma+\tau| \leq k$.

Since $g_{m}$ is assumed to be sufficiently smooth, $\left|\lambda^{\prime}\right| \geq|\lambda|$, and $\partial_{m}^{\tau} \partial_{m}^{\boldsymbol{\beta}_{m}} \psi_{\lambda^{\prime}}^{(m)}$ vanishes when $\left|\tau+\boldsymbol{\beta}_{m}\right| \geq e_{m}$, by invoking (3.2) we see that the worst case occurs when $\rho=0,\left|\tau+\boldsymbol{\beta}_{m}\right|=q:=\min \left(e_{m}-1, k+\left|\boldsymbol{\beta}_{m}\right|\right)$, and thus when $|\sigma|=k-q+\left|\boldsymbol{\beta}_{m}\right|$, yielding

$$
\begin{aligned}
\| 2^{-|\lambda|\left|\boldsymbol{\alpha}_{m}\right|} 2^{-\left|\lambda^{\prime}\right|\left|\boldsymbol{\beta}_{m}\right|} & g_{m} \partial_{m}^{\boldsymbol{\alpha}_{m}} \psi_{\lambda}^{(m)} \partial_{m}^{\boldsymbol{\beta}_{m}} \psi_{\lambda^{\prime}}^{(m)} \|_{W_{\infty}^{k}\left(\Omega_{m}^{\left(l^{\prime}, v^{\prime}\right)}\right)} \\
& \lesssim 2^{\left(|\lambda|+\left|\lambda^{\prime}\right|\right) \frac{d_{m}}{2}} 2^{\left(k-q+\left|\boldsymbol{\beta}_{m}\right|\right)|\lambda|} 2^{\left(q-\left|\boldsymbol{\beta}_{m}\right|\right)\left|\lambda^{\prime}\right|}\left\|g_{m}\right\|_{W_{\infty}^{k}\left(\Omega_{m}^{\left(l^{\prime}, v^{\prime}\right)}\right)}
\end{aligned}
$$

Using the facts that $\operatorname{diam}\left(\Omega_{m}^{\left(l^{\prime}, v^{\prime}\right)}\right) \approx 2^{-\left|\lambda^{\prime}\right|}, \operatorname{vol}\left(\Omega_{m}^{\left(l^{\prime}, v^{\prime}\right)}\right) \approx 2^{-\left|\lambda^{\prime}\right| d_{m}}$, by substituting this result into (5.2), summing over the uniformly bounded number of $\Omega_{m}^{\left(l^{\prime}, v^{\prime}\right)}$ that make up supp $\psi_{\lambda}^{(m)} \cap \operatorname{supp} \psi_{\lambda^{\prime}}^{(m)}$, and by taking $k=k_{m}$ satisfying

$$
k_{m} \geq \max \left(d_{m} a_{m}, b_{m}-\left|\boldsymbol{\beta}_{m}\right|+e_{m}-1\right),
$$

we end up with (5.1).

From now on always taking $b_{m}:=\tilde{p}_{m}+\min \left(\left|\boldsymbol{\alpha}_{m}\right|,\left|\boldsymbol{\beta}_{m}\right|\right) \geq z_{m}^{\left(i_{m}\left(\lambda, \lambda^{\prime}\right)\right.}-\frac{i_{m}\left(\lambda, \lambda^{\prime}\right)}{2}$ $\left(\lambda, \lambda^{\prime} \in \Lambda_{m}\right)$ and the corresponding $k_{m}$ (which also depends on $a_{m}$ ), using (4.5) and (5.1) we have the following result for the standard tensor product quadrature rule:

Lemma 5.1. For $g$ being a function on $\boldsymbol{\Omega}$ with

$$
\boldsymbol{\partial}^{\gamma} g \in L^{\infty}(\boldsymbol{\Omega}) \quad \text { for all }|\gamma| \leq \max (\boldsymbol{k}, \tilde{\boldsymbol{p}}+\min (|\boldsymbol{\alpha}|,|\boldsymbol{\beta}|)),
$$


where $\boldsymbol{k}=\left(k_{1}, \ldots, k_{n}\right)$, we have

$$
\begin{aligned}
& \left|\boldsymbol{I}_{\boldsymbol{\lambda}, \boldsymbol{\lambda}^{\prime}}^{(\boldsymbol{\alpha}, \boldsymbol{\beta})}-\bigotimes_{m=1}^{n} Q_{\boldsymbol{\lambda}_{m}, \boldsymbol{\lambda}_{m}^{\prime}, m, \boldsymbol{N}_{m}}^{\left(\boldsymbol{\alpha}_{m}, \boldsymbol{\beta}_{m}\right)}\right| \lesssim\left(\sum_{m=1}^{n} \boldsymbol{N}_{m}^{-a_{m}}\right) 2^{-|| \boldsymbol{\lambda}|-| \boldsymbol{\lambda}^{\prime}|| \cdot\left(\frac{d}{2}+\boldsymbol{z}^{\left(i\left(\boldsymbol{\lambda}, \boldsymbol{\lambda}^{\prime}\right)\right)}-\frac{i\left(\boldsymbol{\lambda}, \boldsymbol{\lambda}^{\prime}\right)}{2}\right)} \\
& \times \underset{|\boldsymbol{\gamma}| \leq \max (\boldsymbol{k}, \tilde{\boldsymbol{p}}+\min (|\boldsymbol{\alpha}|,|\boldsymbol{\beta}|))}{\max }\left\|\boldsymbol{\partial}^{\gamma} g\right\|_{L^{\infty}\left(\prod_{m=1}^{n} \operatorname{supp} \psi_{\boldsymbol{\lambda}_{m}}^{(m)} \cap \operatorname{supp} \psi_{\boldsymbol{\lambda}_{m}^{\prime}}^{(m)}\right)},
\end{aligned}
$$

where, assuming each evaluation of $g$ takes $\mathcal{O}(1)$ operations, the evaluation of this product rule requires $\mathcal{O}\left(\prod_{m=1}^{n} \boldsymbol{N}_{m}\right)$ operations.

With the optimal choice $\boldsymbol{N}_{m} \approx N^{\frac{1}{a_{m}}\left(\sum_{\ell} \frac{1}{a_{\ell}}\right)^{-1}}$, the number of operations is of or$\operatorname{der} N$, and the error bound is of order $N^{-\left(\sum_{\ell} \frac{1}{a_{\ell}}\right)^{-1}} 2^{-|| \boldsymbol{\lambda}|-| \boldsymbol{\lambda}^{\prime}|| \cdot\left(\frac{d}{2}+\boldsymbol{z}^{\left(i\left(\boldsymbol{\lambda}, \boldsymbol{\lambda}^{\prime}\right)\right)}-\frac{i\left(\boldsymbol{\lambda}, \boldsymbol{\lambda}^{\prime}\right)}{2}\right)}$, that, in case $a_{1}=\ldots=a_{n}=a$, reads as $N^{-a / n} 2^{-|| \boldsymbol{\lambda}|-| \boldsymbol{\lambda}^{\prime} \mid \cdot \cdot\left(\frac{d}{2}+\boldsymbol{z}^{\left(i\left(\boldsymbol{\lambda}, \boldsymbol{\lambda}^{\prime}\right)\right)}-\frac{i\left(\boldsymbol{\lambda}, \boldsymbol{\lambda}^{\prime}\right)}{2}\right)}$.

Proof. Dropping the indices $\boldsymbol{\lambda}_{m}, \boldsymbol{\lambda}_{m}^{\prime}, \boldsymbol{N}_{m}$, and $\left(\boldsymbol{\alpha}_{m}, \boldsymbol{\beta}_{m}\right)$, using induction the proof follows easily by writing

$$
\begin{aligned}
& I_{1} \otimes \cdots \otimes I_{n}-Q_{1} \otimes \cdots \otimes Q_{n}=\left(I_{1} \otimes \cdots \otimes I_{n-1}-Q_{1} \otimes \cdots \otimes Q_{n-1}\right) \otimes I_{n} \\
& +I_{1} \otimes \cdots \otimes I_{n-1} \otimes\left(I_{n}-Q_{n}\right)-\left(I_{1} \otimes \cdots \otimes I_{n-1}-Q_{1} \otimes \cdots \otimes Q_{n-1}\right) \otimes\left(I_{n}-Q_{n}\right) .
\end{aligned}
$$

In Lemma 5.1 we see an instance of the "curse of dimensionality": the rate of convergence of the tensor product quadrature formula as a function of the number of quadrature points $N$ is inversely proportional to $n$. If the tensor product quadrature rules are applied for the approximation of the entries $\boldsymbol{I}_{\boldsymbol{\lambda}, \boldsymbol{\lambda}^{\prime}}^{(\boldsymbol{\alpha}, \boldsymbol{\beta})}$, then in order to show $s^{*}$-computability of $\boldsymbol{I}^{(\boldsymbol{\alpha}, \boldsymbol{\beta})}$, the parameters $a_{m}$, i.e., the orders of the composite rules, and thus at the same time, the orders of the partial derivatives of $g$ that are required to be bounded, should increase proportionally with $n$. As we will now see, this curse of dimensionality can be avoided by applying sparse tensor product rules introduced in Smo63; see also GG98.

Lemma 5.2. For $1 \leq m \leq n$, let $\left(N_{j}^{(m)}\right)_{j \in \mathbb{N}} \subset \mathbb{N}$ be a sequence such that $C \geq N_{j+1}^{(m)} / N_{j}^{(m)} \geq c$ for some absolute constants $C \geq c>1$. Then under the assumptions of Lemma [5.1, and with $Q_{\lambda, \lambda^{\prime}, m, N_{-1}^{(m)}}^{(\alpha, \beta)}:=0$, for any $N \in \mathbb{N}$ the sparse tensor product quadrature rule

$$
\boldsymbol{Q}_{\boldsymbol{\lambda}, \boldsymbol{\lambda}^{\prime}, N}^{(\boldsymbol{\alpha}, \boldsymbol{\beta})}:=\sum_{\left\{\boldsymbol{j} \in \mathbb{N}^{n}: \prod_{m=1}^{n}\left(N_{\boldsymbol{j}_{m}}^{(m)}\right)^{a_{m}} \leq N^{\left.m^{m_{\ell} a_{\ell}}\right\}}\right.} \bigotimes_{m=1}^{n}\left(Q_{\boldsymbol{\lambda}_{m}, \boldsymbol{\lambda}_{m}^{\prime}, m, N_{\boldsymbol{j}_{m}}^{(m)}}^{\left(\boldsymbol{\alpha}_{m}, \boldsymbol{\beta}_{m}\right)}-Q_{\boldsymbol{\lambda}_{m}, \boldsymbol{\lambda}_{m}^{\prime}, m, N_{\boldsymbol{j}_{m}-1}^{(m)}}^{\left(\boldsymbol{\alpha}_{m}, \boldsymbol{\beta}_{m}\right)}\right)
$$

satisfies

$$
\begin{aligned}
\left|\boldsymbol{I}_{\boldsymbol{\lambda}, \boldsymbol{\lambda}^{\prime}}^{(\boldsymbol{\alpha}, \boldsymbol{\beta})}-\boldsymbol{Q}_{\boldsymbol{\lambda}, \boldsymbol{\lambda}^{\prime}, N}^{(\boldsymbol{\alpha}, \boldsymbol{\beta})}\right| \lesssim & (\log N)^{n-1} N^{-\min _{\ell} a_{\ell} 2^{-}|| \boldsymbol{\lambda}|-| \boldsymbol{\lambda}^{\prime}|| \cdot\left(\frac{d}{2}+\boldsymbol{z}^{\left(i\left(\boldsymbol{\lambda}, \boldsymbol{\lambda}^{\prime}\right)\right)}-\frac{i\left(\boldsymbol{\lambda}, \boldsymbol{\lambda}^{\prime}\right)}{2}\right)} \\
& \times \max _{|\boldsymbol{\gamma}| \leq \max (\boldsymbol{k}, \tilde{\boldsymbol{p}}+\min (|\boldsymbol{\alpha}|,|\boldsymbol{\beta}|))}\left\|\boldsymbol{\partial}^{\boldsymbol{\gamma}} g\right\|_{L^{\infty}\left(\prod_{m=1}^{n} \operatorname{supp} \psi_{\boldsymbol{\lambda}_{m}}^{(m)} n \operatorname{supp} \psi_{\boldsymbol{\lambda}_{m}^{\prime}}^{(m)}\right)}
\end{aligned}
$$

where the evaluation of this rule requires $\mathcal{O}\left(N(\log N)^{n-1}\right)$ operations.

Proof. In this proof, we drop the indices $\boldsymbol{\lambda}_{m}, \boldsymbol{\lambda}_{m}^{\prime}, \boldsymbol{\alpha}_{m}$ and $\boldsymbol{\beta}_{m}$. In view of Lemma 5.1 , the quadrature error can be written as

$$
\sum_{\left\{\boldsymbol{j} \in \mathbb{N}^{n}: \prod_{m=1}^{n}\left(N_{\boldsymbol{j}_{m}}^{(m)}\right)^{a_{m}}>N^{\left.\min _{\ell} a_{\ell}\right\}}\right.} \bigotimes_{m=1}^{n}\left(Q_{m, N_{\boldsymbol{j}_{m}}^{(m)}}-Q_{m, N_{\boldsymbol{j}_{m}-1}^{(m)}}\right) .
$$


By the convention $Q_{m, N_{\boldsymbol{j}_{m}-1}^{(m)}}=0$ for $\boldsymbol{j}_{m}=0$, writing $Q_{m, N_{0}^{(m)}}=I_{m}+Q_{m, N_{0}^{(m)}}-I_{m}$, from (4.5) and (5.1), we infer that the error, in modulus, can be bounded by some absolute multiple of

$$
\begin{aligned}
& 2^{-|| \boldsymbol{\lambda}|-| \boldsymbol{\lambda}^{\prime}|| \cdot\left(\frac{d}{2}+\boldsymbol{z}^{\left(i\left(\boldsymbol{\lambda}, \boldsymbol{\lambda}^{\prime}\right)\right)}-\frac{i\left(\boldsymbol{\lambda}, \boldsymbol{\lambda}^{\prime}\right)}{2}\right)} \sum_{\left\{\boldsymbol{j} \in \mathbb{N}^{n}: \prod_{m=1}^{n}\left(N_{\boldsymbol{j}_{m}}^{(m)}\right)^{a_{m}}>N^{\left.\min _{\ell} a_{\ell}\right\}}\right.} \prod_{m=1}^{n}\left(N_{\boldsymbol{j}_{m}}^{(m)}\right)^{-a_{m}} \\
& \bar{\approx} 2^{-|| \boldsymbol{\lambda}|-| \boldsymbol{\lambda}^{\prime}|| \cdot\left(\frac{d}{2}+\boldsymbol{z}^{\left(i\left(\boldsymbol{\lambda}, \boldsymbol{\lambda}^{\prime}\right)\right)}-\frac{i\left(\boldsymbol{\lambda}, \boldsymbol{\lambda}^{\prime}\right)}{2}\right)}(\log N)^{n-1} N^{-\min _{\ell} a_{\ell}}
\end{aligned}
$$

times $\max _{|\boldsymbol{\gamma}| \leq \max (\boldsymbol{k}, \tilde{\boldsymbol{p}}+\min (|\boldsymbol{\alpha}|,|\boldsymbol{\beta}|))}\left\|\boldsymbol{\partial}^{\gamma} g\right\|_{L^{\infty}(\ldots)}$. The work can be bounded by some absolute multiple of

$$
\begin{aligned}
\sum_{\left\{\boldsymbol{j} \in \mathbb{N}^{n}: \prod_{m=1}^{n}\left(N_{\boldsymbol{j}_{m}}^{(m)}\right)^{a_{m}} \leq N^{\left.\min _{\ell} a_{\ell}\right\}}\right.} \prod_{m=1}^{n} N_{\boldsymbol{j}_{m}}^{(m)} & \leq \sum_{\left\{\boldsymbol{j} \in \mathbb{N}^{n}: \prod_{m=1}^{n} N_{\boldsymbol{j}_{m}}^{(m)} \leq N\right\}} \prod_{m=1}^{n} N_{\boldsymbol{j}_{m}}^{(m)} \\
& \lesssim(\log N)^{n-1} N .
\end{aligned}
$$

\section{Computability}

We now turn to our main result, the $s^{*}$-computability of an approximation of the matrix $\boldsymbol{I}^{(\boldsymbol{\alpha}, \boldsymbol{\beta})}$.

Lemma 6.1. As in Lemma 4.2, for some $\boldsymbol{w} \in(0, \infty)^{n}, \bar{s}>0$, and any $q \in \mathbb{N}$, let $\boldsymbol{B}_{q}=\left(\left(\boldsymbol{B}_{q}\right)_{\boldsymbol{\lambda}, \boldsymbol{\lambda}^{\prime}}\right)_{\boldsymbol{\lambda}, \boldsymbol{\lambda}^{\prime} \in \boldsymbol{\Lambda}}$ be a matrix, partitioned into blocks

$$
\left(\boldsymbol{B}_{q}\right)_{\boldsymbol{l}, \boldsymbol{l}^{\prime}}=\left(\left(\boldsymbol{B}_{q}\right)_{\boldsymbol{\lambda}, \boldsymbol{\lambda}^{\prime}}\right)_{|\boldsymbol{\lambda}|=\boldsymbol{l},\left|\boldsymbol{\lambda}^{\prime}\right|=\boldsymbol{l}^{\prime}},
$$

such that the maximal number of non-zero entries in each row of $\left(\boldsymbol{B}_{q}\right)_{\boldsymbol{l}, \boldsymbol{l}^{\prime}}$ or column of $\left(\boldsymbol{B}_{q}\right)_{\boldsymbol{l}^{\prime}, \boldsymbol{l}}$ is bounded by some absolute multiple of $2^{\max \left(\boldsymbol{l}^{\prime}-\boldsymbol{l}, 0\right) \cdot \boldsymbol{w} / \bar{s}}$, and

$$
\left(\boldsymbol{B}_{q}\right)_{\boldsymbol{l}, \boldsymbol{l}^{\prime}}=0 \text { when }\left|\boldsymbol{l}^{\prime}-\boldsymbol{l}\right| \cdot \boldsymbol{w}>q .
$$

For some constants $\sigma, \tau>0, v, w \geq 0$, and any $N \in \mathbb{N}$, suppose we have a numerical approximation $\left(\boldsymbol{B}_{q}\right)_{\boldsymbol{\lambda}, \boldsymbol{\lambda}^{\prime}, N}$ to $\left(\boldsymbol{B}_{q}\right)_{\boldsymbol{\lambda}, \boldsymbol{\lambda}^{\prime}}$, whose computation requires $\mathcal{O}\left(N(\log N)^{v}\right)$ operations, such that

$$
\left|\left(\boldsymbol{B}_{q}\right)_{\boldsymbol{\lambda}, \boldsymbol{\lambda}^{\prime}}-\left(\boldsymbol{B}_{q}\right)_{\boldsymbol{\lambda}, \boldsymbol{\lambda}^{\prime}, N}\right| \lesssim N^{-\sigma}(\log N)^{w} 2^{-\frac{1 / 2+\tau}{\bar{s}}}|| \boldsymbol{\lambda}^{\prime}|-| \boldsymbol{\lambda}|| \cdot \boldsymbol{w} .
$$

Then with $\boldsymbol{B}_{q}^{*}$ being defined by replacing any entry $\left(\boldsymbol{B}_{q}\right)_{\boldsymbol{\lambda}, \boldsymbol{\lambda}^{\prime}}$ of $\boldsymbol{B}_{q}$ with $\left|\boldsymbol{l}^{\prime}-\boldsymbol{l}\right| \cdot \boldsymbol{w} \leq q$ by $\left(\boldsymbol{B}_{q}\right)_{\boldsymbol{\lambda}, \boldsymbol{\lambda}^{\prime}, N(q)_{\boldsymbol{\lambda}, \boldsymbol{\lambda}^{\prime}}}$, where

$$
N(q)_{\boldsymbol{\lambda}, \boldsymbol{\lambda}^{\prime}} \approx 2^{\left(q-|| \boldsymbol{\lambda}^{\prime}|-| \boldsymbol{\lambda}|| \cdot \boldsymbol{w}\right) / \bar{s}},
$$

the work for computing each row or column of $\boldsymbol{B}_{q}^{*}$ is bounded by $\mathcal{O}\left(q^{n+v} 2^{q / \bar{s}}\right)$ and $\left\|\boldsymbol{B}_{q}-\boldsymbol{B}_{q}^{*}\right\| \lesssim q^{n+w} 2^{-\min (\sigma, \tau) q / \bar{s}}$.

Proof. To prove the statement concerning the amount of work, it is sufficient to bound the number of operations needed for computing the entries $\left(\boldsymbol{B}_{q}^{*}\right)_{\boldsymbol{\lambda}, \boldsymbol{\lambda}^{\prime}}$ for any fixed $\boldsymbol{\lambda}$, and all $\boldsymbol{\lambda}^{\prime}$ with $\left|\boldsymbol{\lambda}^{\prime}\right| \geq|\boldsymbol{\lambda}|$. This number can be bounded by some absolute multiple of

$$
q^{v} \sum_{\left\{\boldsymbol{\lambda}^{\prime}: 0 \leq\left(\left|\boldsymbol{\lambda}^{\prime}\right|-|\boldsymbol{\lambda}|\right) \cdot \boldsymbol{w} \leq q\right\}} 2^{\left(q-\left(\left|\boldsymbol{\lambda}^{\prime}\right|-|\boldsymbol{\lambda}|\right) \cdot \boldsymbol{w}\right) / \bar{s}} \lesssim q^{v} 2^{q / \bar{s}} \sum_{\left\{\boldsymbol{l}^{\prime}: 0 \leq\left(\boldsymbol{l}^{\prime}-|\boldsymbol{\lambda}|\right) \cdot \boldsymbol{w} \leq q\right\}} 1 \lesssim q^{n+v} 2^{q / \bar{s}} .
$$


Consider the block $\boldsymbol{E}_{\boldsymbol{l}, \boldsymbol{l}^{\prime}}=\left(\left(\boldsymbol{B}_{q}\right)_{\boldsymbol{\lambda}, \boldsymbol{\lambda}^{\prime}}-\left(\boldsymbol{B}_{q}^{*}\right)_{\boldsymbol{\lambda}, \boldsymbol{\lambda}^{\prime}}\right)_{|\boldsymbol{\lambda}|=\boldsymbol{l},\left|\boldsymbol{\lambda}^{\prime}\right|=\boldsymbol{l}^{\prime}}$. Bounding the squared norm of $\boldsymbol{E}_{\boldsymbol{l}, \boldsymbol{l}^{\prime}}$ by the product of its maximal absolute row-sum and its maximal absolute column-sum, we find that

$$
\begin{aligned}
\left\|\boldsymbol{E}_{\boldsymbol{l}, \boldsymbol{l}^{\prime}}\right\| & \lesssim 2^{\frac{1}{2}\left|\boldsymbol{l}^{\prime}-\boldsymbol{l}\right| \cdot \boldsymbol{w} / \bar{s}} q^{w} 2^{-\frac{\sigma}{\bar{s}}\left(q-\left|\boldsymbol{l}^{\prime}-\boldsymbol{l}\right| \cdot \boldsymbol{w}\right)} 2^{-\frac{\frac{1}{2}+\tau}{\bar{s}}\left|\boldsymbol{l}^{\prime}-\boldsymbol{l}\right| \cdot \boldsymbol{w}} \\
& =q^{w} 2^{-\sigma q / \bar{s}} 2^{\frac{\sigma-\tau}{\bar{s}}\left|\boldsymbol{l}^{\prime}-\boldsymbol{l}\right| \cdot \boldsymbol{w}} .
\end{aligned}
$$

Bounding $\left\|\boldsymbol{B}_{q}-\boldsymbol{B}_{q}^{*}\right\|^{2}$ on $\max _{\boldsymbol{l}} \sum_{\left\{\boldsymbol{l}^{\prime}:\left|\boldsymbol{l}^{\prime}-\boldsymbol{l}\right| \cdot \boldsymbol{w} \leq q\right\}}\left\|\boldsymbol{E}_{\boldsymbol{l}, \boldsymbol{l}^{\prime}}\right\| \times \max _{\boldsymbol{l}^{\prime}} \sum_{\left\{\boldsymbol{l}:\left|\boldsymbol{l}^{\prime}-\boldsymbol{l}\right| \cdot \boldsymbol{w} \leq q\right\}}\left\|\boldsymbol{E}_{\boldsymbol{l}, \boldsymbol{l}^{\prime}}\right\|$, which is $\mathcal{O}\left(\left(q^{w} 2^{-\sigma q / \bar{s}}\right)^{2}\right), \mathcal{O}\left(\left(q^{w} q^{n} 2^{-\sigma q / \bar{s}}\right)^{2}\right)$, or $\mathcal{O}\left(\left(q^{w} q^{n-1} 2^{-\tau q / \bar{s}}\right)^{2}\right)$, for $\sigma<\tau$, $\sigma=\tau$, or $\sigma>\tau$, respectively, the proof is completed.

Theorem 6.2. Let $\boldsymbol{\alpha}, \boldsymbol{\beta}, \boldsymbol{I}^{(\boldsymbol{\alpha}, \boldsymbol{\beta})}, \boldsymbol{I}_{q}^{(\boldsymbol{\alpha}, \boldsymbol{\beta})}, \boldsymbol{z}^{(\boldsymbol{i})}$, and $s^{*}$ be as in Theorem 4.1. Assume that for $1 \leq m \leq n$, (5.1) is valid with $a_{m}=s^{*}$ and $b_{m}=\tilde{p}_{m}+\min \left(\left|\boldsymbol{\alpha}_{m}\right|,\left|\boldsymbol{\beta}_{m}\right|\right)$, and that for the corresponding $k_{m}$ and with $\mathbf{k}:=\left(k_{1}, \ldots, k_{n}\right)$,

$$
\partial^{\gamma} g \in L^{\infty}(\boldsymbol{\Omega}), \quad|\gamma| \leq \max (\boldsymbol{k}, \tilde{\boldsymbol{p}}+\min (|\boldsymbol{\alpha}|,|\boldsymbol{\beta}|))
$$

Denote for $\boldsymbol{i} \in\{0,1\}^{n}$,

$$
\boldsymbol{w}^{(i)}:=\frac{s^{*}}{s^{*}+1 / 2}\left(\frac{d}{2}+\boldsymbol{z}^{(i)}-\frac{i}{2}\right)
$$

We define for $q \in \mathbb{N}$ the approximation $\boldsymbol{I}_{q}^{*(\boldsymbol{\alpha}, \boldsymbol{\beta})}$ for $\boldsymbol{I}_{q}^{(\boldsymbol{\alpha}, \boldsymbol{\beta})}$ approximating $\boldsymbol{I}_{\boldsymbol{\lambda}, \boldsymbol{\lambda}^{\prime}}^{(\boldsymbol{\alpha}, \boldsymbol{\beta})}$ for || $\boldsymbol{\lambda}^{\prime}|-| \boldsymbol{\lambda} \| \cdot \boldsymbol{z}^{\left(\boldsymbol{i}\left(\boldsymbol{\lambda}, \boldsymbol{\lambda}^{\prime}\right)\right)} \leq q$ by the sparse product rule $\boldsymbol{Q}_{\boldsymbol{\lambda}, \boldsymbol{\lambda}^{\prime}, N(q)_{\boldsymbol{\lambda}, \boldsymbol{\lambda}^{\prime}}}^{(\boldsymbol{\alpha}, \boldsymbol{\beta})}$ with

$$
N(q)_{\boldsymbol{\lambda}, \boldsymbol{\lambda}^{\prime}} \approx 2^{\left(q-|| \boldsymbol{\lambda}^{\prime}|-| \boldsymbol{\lambda}|| \cdot \boldsymbol{w}^{\left(i\left(\boldsymbol{\lambda}, \boldsymbol{\lambda}^{\prime}\right)\right)}\right) / s^{*}}
$$

Then the work for computing each row or column of $\boldsymbol{I}_{q}^{*(\boldsymbol{\alpha}, \boldsymbol{\beta})}$ subject to the global accuracy constraint $\left\|\boldsymbol{I}^{(\boldsymbol{\alpha}, \boldsymbol{\beta})}-\boldsymbol{I}_{q}^{*(\boldsymbol{\alpha}, \boldsymbol{\beta})}\right\| \lesssim q^{2 n-1} 2^{-q}$, can be bounded by $\mathcal{O}\left(q^{2 n-1} 2^{q / s^{*}}\right)$.

In particular, $\boldsymbol{I}^{(\boldsymbol{\alpha}, \boldsymbol{\beta})}$ is $\mathrm{s}^{*}$-computable.

Proof. As shown in Lemma 5.2, we have

$$
\begin{aligned}
\left|\boldsymbol{I}_{\boldsymbol{\lambda}, \boldsymbol{\lambda}^{\prime}}^{(\boldsymbol{\alpha}, \boldsymbol{\beta})}-\boldsymbol{Q}_{\boldsymbol{\lambda}, \boldsymbol{\lambda}^{\prime}, N}^{(\boldsymbol{\alpha}, \boldsymbol{\beta})}\right| & \lesssim(\log N)^{n-1} N^{-s^{*}} 2^{-|| \boldsymbol{\lambda}|-| \boldsymbol{\lambda}^{\prime}|| \cdot\left(\frac{\boldsymbol{d}}{2}+\boldsymbol{z}^{\left(i\left(\boldsymbol{\lambda}, \boldsymbol{\lambda}^{\prime}\right)\right)}-\frac{i\left(\boldsymbol{\lambda}, \boldsymbol{\lambda}^{\prime}\right)}{2}\right)} \\
& =(\log N)^{n-1} N^{-s^{*}} 2^{-\frac{1 / 2+s^{*}}{s^{*}}|| \boldsymbol{\lambda}|-| \boldsymbol{\lambda}^{\prime}|| \cdot \boldsymbol{w}^{\left(i\left(\boldsymbol{\lambda}, \boldsymbol{\lambda}^{\prime}\right)\right)}}
\end{aligned}
$$

with the evaluation of $\boldsymbol{Q}_{\boldsymbol{\lambda}, \boldsymbol{\lambda}^{\prime}, N}^{(\boldsymbol{\alpha}, \boldsymbol{\beta})}$ taking $\mathcal{O}\left(N(\log N)^{n-1}\right)$ operations. Suppressing the dependence on $(\boldsymbol{\alpha}, \boldsymbol{\beta})$ in the notations, we write $\boldsymbol{I}=\sum_{\boldsymbol{i} \in\{0,1\}^{n}} \boldsymbol{I}^{(\boldsymbol{i})}, \boldsymbol{I}_{q}=$ $\sum_{\boldsymbol{i} \in\{0,1\}^{n}} \boldsymbol{I}_{q}^{(\boldsymbol{i})}$, and $\boldsymbol{I}_{q}^{*}=\sum_{\boldsymbol{i} \in\{0,1\}^{n}} \boldsymbol{I}_{q}^{*(\boldsymbol{i})}$, where analogously to $\boldsymbol{I}^{(\boldsymbol{i})}$ and $\boldsymbol{I}_{q}^{(\boldsymbol{i})}, \boldsymbol{I}_{q}^{*(\boldsymbol{i})}$ contains all non-zero entries of $\boldsymbol{I}_{q}^{*}$ with indices $\boldsymbol{\lambda}, \boldsymbol{\lambda}^{\prime}$ with $\boldsymbol{i}\left(\boldsymbol{\lambda}, \boldsymbol{\lambda}^{\prime}\right)=\boldsymbol{i}$. Recall that the non-zero part of $\boldsymbol{I}_{q}^{(i)}$ consists of those blocks $\boldsymbol{I}_{\boldsymbol{l}, \boldsymbol{l}^{\prime}}^{(\boldsymbol{i})}=\left(\boldsymbol{I}_{\boldsymbol{\lambda}, \boldsymbol{\lambda}^{\prime}}^{(\boldsymbol{i})}\right)_{|\boldsymbol{\lambda}|=\boldsymbol{l},\left|\boldsymbol{\lambda}^{\prime}\right|=\boldsymbol{l}^{\prime}}$ with $\left|\boldsymbol{l}^{\prime}-\boldsymbol{l}\right| \cdot \boldsymbol{z}^{(\boldsymbol{i})} \leq q$, and that the number of non-zero entries in each row of $\boldsymbol{I}_{\boldsymbol{l}, \boldsymbol{l}^{\prime}}^{(\boldsymbol{i})}$ or column of $\boldsymbol{I}_{\boldsymbol{l}^{\prime}, \boldsymbol{l}}^{(\boldsymbol{i})}$ can be bounded on some absolute multiple of $2^{\max \left(\boldsymbol{l}^{\prime}-\boldsymbol{l}, 0\right) \cdot(\boldsymbol{d}-\boldsymbol{i})}$.

By definition of $s^{*}$, we have $\boldsymbol{z}^{(\boldsymbol{i})} \geq s^{*}(\boldsymbol{d}-\boldsymbol{i})$, which inequality is equivalent to both $(\boldsymbol{d}-\boldsymbol{i}) \leq \boldsymbol{w}^{(\boldsymbol{i})} / s^{*}$ and $\boldsymbol{z}^{(\boldsymbol{i})} \geq \boldsymbol{w}^{(\boldsymbol{i})}$. Now the proof is completed by an application, for each $\boldsymbol{i} \in\{0,1\}^{n}$, of Lemma 6.1 with $\left(\boldsymbol{B}_{q}, \boldsymbol{B}_{q}^{*}\right)$ reading as $\left(\boldsymbol{I}_{q}^{(\boldsymbol{i})}, \boldsymbol{I}_{q}^{*(\boldsymbol{i})}\right)$. 


\section{Conclusion}

With $s^{*}$ from (4.4), we have shown $s^{*}$-computability of the infinite stiffness matrix of $\partial^{\beta} g \partial^{\alpha}$ with respect to suitable tensor product wavelet bases. As exposed in Sect. 1.3. 2.2. and 2.3, as a consequence the stiffness matrices resulting from Galerkin discretizations of scalar PDEs using the tensorized wavelet bases (1.4), and that of a system of PDEs resulting from an $n$-scale homogenization problem are $s^{*}$-computable, and the stiffness matrix of an anisotropic problem is uniformly $s^{*}$-computable, all for sufficiently large values of $s^{*}$. As a result, adaptive wavelet algorithms for solving these problems converge with the dimension independent rates of best $N$-term approximations in linear complexity. The dependence on $n$ of the constant involved in the error bound for the approximations produced by the adaptive wavelet algorithm, and actually the reduction of this dependence, will be the topic of our future investigations in this field.

\section{REFERENCES}

[AB96] G. Allaire and M. Briane. Multiscale convergence and reiterated homogenization. Proc. Roy. Soc. Edinburgh, 126A:297-342, 1996. MR1386865 (97d:35014)

[Al192] G. Allaire. Homogenization and two scale convergence. SIAM J. Applied Mathematics, 23:1482-1518, 1992. MR1185639 (93k:35022)

[BLP78] A. Bensoussan, J.L. Lions, and G. Papanicolau. Asymptotic Analysis for Periodic Structures. North-Holland Publishing, Amsterdam, 1978. MR0503330 (82h:35001)

[BSS04] S. Beuchler, R. Schneider, and Ch. Schwab. Multiresolution weighted norm equivalences and applications. Numer. Math., 98:67-97, 2004. MR2076054 (2005g:65199)

[CTU99] C. Canuto, A. Tabacco, and K. Urban. The wavelet element method part I: Construction and analysis. Appl. Comput. Harmon. Anal., 6:1-52, 1999. MR 1664902 (99k:42055)

[CDD01] A. Cohen, W. Dahmen, and R. DeVore. Adaptive wavelet methods for elliptic operator equations - Convergence rates. Math. Comp, 70:27-75, 2001. MR.1803124(2002h:65201)

[CDD02] A. Cohen, W. Dahmen, and R. DeVore. Adaptive wavelet methods II - Beyond the elliptic case. Found. Comput. Math., 2(3):203-245, 2002. MR1907380 (2003f:65212)

[Coh03] A. Cohen. Numerical Analysis of Wavelet Methods. Elsevier, Amsterdam, 2003. MR.1990555 (2004c:65178)

[DKU99] W. Dahmen, A. Kunoth, and K. Urban. Biorthogonal spline-wavelets on the interval - Stability and moment conditions. Appl. Comp. Harm. Anal., 6:132-196, 1999. MR:1676771 (99m:42046)

[DS99a] W. Dahmen and R. Schneider. Composite wavelet bases for operator equations. Math. Comp., 68:1533-1567, 1999. MR1648379(99m:65122)

[DS99b] W. Dahmen and R. Schneider. Wavelets on manifolds I: Construction and domain decomposition. SIAM J. Math. Anal., 31:184-230, 1999. MR.1742299(2000k:65242)

[DS99c] W. Dahmen and R.P. Stevenson. Element-by-element construction of wavelets satisfying stability and moment conditions. SIAM J. Numer. Anal., 37(1):319-352, 1999. MR1742747 (2001c:65144)

[DeV98] R. DeVore. Nonlinear approximation. Acta Numer., 7:51-150, 1998 . MR.1689432 (2001a:41034)

[Gan06] T. Gantumur. An optimal adaptive wavelet method for nonsymmetric and indefinite elliptic problems. Technical Report 1343, Utrecht University, January 2006. Submitted.

[GHS05] T. Gantumur, H. Harbrecht, and R.P. Stevenson. An optimal adaptive wavelet method without coarsening of the iterands. Technical Report 1325, Utrecht University, March 2005. To appear in Math. Comp.

[GS04] T. Gantumur and R.P. Stevenson. Computation of differential operators in wavelet coordinates. Math. Comp., 75:697-709, 2006. MR.2196987

[GG98] T. Gerstner and M. Griebel. Numerical integration using sparse grids. Numer. Algorithms, 18:209-232, 1998. MR 1669959 (99m:65042)

[GK00] M. Griebel and S. Knapek. Optimized tensor-product approximation spaces. Constr. Approx., 16(4):525-540, 2000. MR1771694 (2001g:41025) 
[GO95] M. Griebel and P. Oswald. Tensor product type subspace splittings and multilevel iterative methods for anisotropic problems. Adv. Comput. Math., 4(1-2):171-206, 1995. MR1338900 (96e:65069)

[HS04] H. Harbrecht and R.P. Stevenson. Wavelets with patchwise cancellation properties. Math. Comp., 75:1871-1889, 2006. MR2240639

[HMS05] N. Hilber, A.M. Matache, and Ch. Schwab. Sparse wavelet methods for option pricing under stochastic volatility. The Journal of Computational Finance, 8(4):1-42, 2005.

[HS05] V.H. Hoang and Ch. Schwab. High dimensional finite elements for elliptic problems with multiple scales. Multiscale Modeling and Simulation: A SIAM Interdisciplinary Journal, 3:168-194, 2005. MR2123115 (2005m:65267)

[LC85] W. A. Light and E. W. Cheney. Approximation theory in tensor product spaces, volume 1169 of Lecture Notes in Mathematics. Springer-Verlag, Berlin, 1985. MR817984 (87g:41064)

[Nit06] P. A. Nitsche. Best $N$-term approximation spaces for tensor product wavelet bases. Const. Approx., 24(1), 49-70, 2006. MR2217525(2007a:41032)

[vPS04] T. von Petersdorff and Ch. Schwab. Numerical solution of parabolic problems in high dimensions. M2AN Mathematical Modelling and Numerical Analysis, 38:93-121, 2004. MR2073932(2005d:65169)

[vPS06] T. von Petersdorff and Ch. Schwab. Sparse finite element methods for operator equations with stochastic data. Applications of Mathematics, 45:145-180, 2006. MR.2212311

[Pri06] M. Primbs. Stabile biorthogonale Spline-Waveletbasen auf dem Intervall. Ph.D. thesis, Universität Duisburg, 2006.

[Smo63] S.A. Smolyak. Quadrature and interpolation formulas for tensor products of certain classes of functions. Dokl. Akad. Nauk SSSR, 4:240-243, 1963.

[Ste04] R.P. Stevenson. Composite wavelet bases with extended stability and cancellation properties. SIAM J. Numer. Anal., 45(2):133-162, 2007.

Seminar for Applied Mathematics, ETHZ HG G58.1, ETH Zürich, CH 8092 Zürich, SWITZERLAND

E-mail address: schwab@sam.math.ethz.ch

Department of Mathematics, Utrecht University, P.O. Box 80.010, NL-3508 TA Utrecht, The NetherLANDS

E-mail address: stevenson@math.uu.nl 